\title{
Stability in orbit equivalence for Baumslag-Solitar groups and Vaes groups
}

\author{
Yoshikata Kida
}

\begin{abstract}
A measure-preserving action of a discrete countable group on a standard probability space is called stable if the associated equivalence relation is isomorphic to its direct product with the ergodic hyperfinite equivalence relation of type $\mathrm{II}_{1}$. We show that any Baumslag-Solitar group has such an ergodic, free and stable action. It follows that any Baumslag-Solitar group is measure equivalent to its direct product with any amenable group. The same property is obtained for the inner amenable groups of Vaes.
\end{abstract}

Mathematics Subject Classification (2010). 20E06, 20E08, 37A20.

Keywords. Baumslag-Solitar groups, Vaes groups, stability, measure equivalence, orbit equivalence.

\section{Contents}

1 Introduction . . . . . . . . . . . . . . . . . . . . . 204

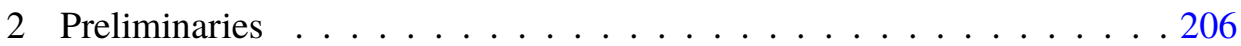

3 Actions of certain solvable quotients . . . . . . . . . . . . . . 209

4 Stable actions of Baumslag-Solitar groups . . . . . . . . . . . 216

5 Semi-direct products ME to direct products . . . . . . . . . . 228

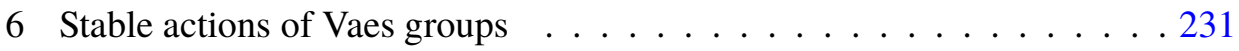

References . . . . . . . . . . . . . . . . . . . . . . . . 234 


\section{Introduction}

We mean by a p.m.p. action of a discrete countable group a measure-preserving action of the group on a standard Borel space equipped with a probability measure, where "p.m.p." stands for "probability-measure-preserving." A p.m.p. action of a discrete countable group is called stable if the associated equivalence relation is isomorphic to its direct product with the ergodic hyperfinite equivalence relation of type $\mathrm{II}_{1}$. Due to Connes, Feldman and Weiss [4] and to Ornstein and Weiss [19], any ergodic, free and p.m.p. action of any infinite amenable group gives rise to the ergodic hyperfinite equivalence relation of type $\mathrm{II}_{1}$. It is a challenging problem to decide whether a given group has an ergodic, free, p.m.p. and stable action unless the group virtually has a direct summand which is infinite and amenable.

Jones and Schmidt [15] characterized stability of ergodic p.m.p. actions in terms of asymptotically central sequences. In [15, Example 4.4], they also noticed that for any collection of countably infinitely many, non-trivial discrete countable groups, $\left\{G_{n}\right\}_{n}$, and for any ergodic, free and p.m.p. action $G_{n} \curvearrowright\left(X_{n}, \mu_{n}\right)$, the product action of the direct sum, $\bigoplus_{n} G_{n} \curvearrowright \prod_{n}\left(X_{n}, \mu_{n}\right)$, is stable.

On the other hand, Zimmer [24] obtained certain indecomposability results on equivalence relations arising from semisimple Lie groups. Adams [1] showed that for any ergodic, free and p.m.p. action of a non-elementary word-hyperbolic group, the associated equivalence relation cannot be written as the direct product of two discrete measured equivalence relations of type $\mathrm{II}_{1}$. Such indecomposability is also obtained for any equivalence relation with its cost more than 1 or its first $\ell^{2}$-Betti number positive in [10] and [11], and for the equivalence relation associated with any action of the mapping class group of a surface in [16]. More strongly, the von Neumann algebras associated with various group actions are shown to be prime in [2], [3], [13], [14], [20], and [21].

For two integers $p, q$ with $1 \leq|p| \leq|q|$, the group with the presentation,

$$
\mathrm{BS}(p, q)=\left\langle a, t \mid t a^{p} t^{-1}=a^{q}\right\rangle,
$$

is called the Baumslag-Solitar group. The group $\mathrm{BS}(p, q)$ is amenable if and only if $|p|=1$. If $2 \leq|p|=|q|$, then $\mathrm{BS}(p, q)$ has a finite index subgroup isomorphic to the direct product of the infinite cyclic group $\mathbb{Z}$ with a non-abelian free group of finite rank. It readily follows that there exists a free and stable action of $\operatorname{BS}(p, q)$ unless $2 \leq|p|<|q|$. If $2 \leq|p|<|q|$, then no finite index subgroup of $\operatorname{BS}(p, q)$ has an infinite, amenable and normal subgroup. This is proved through the action of $\mathrm{BS}(p, q)$ on the Bass-Serre tree and its boundary, as discussed in the first paragraph of [17, Appendix B]. We could expect Adams' argument in [1] applicable 
to $\mathrm{BS}(p, q)$ because the action of a word-hyperbolic group on its compactification plays an important role in his proof. By contrast, applying Jones-Schmidt's characterization of stable actions, we show the following:

Theorem 1.1. Let $p$ and $q$ be integers with $2 \leq|p|<|q|$. Then $\mathrm{BS}(p, q)$ has an ergodic, free, p.m.p. and stable action. In particular, for any amenable, discrete and countable group $A$, the group $\mathrm{BS}(p, q)$ is measure equivalent to the direct product $A \times \mathrm{BS}(p, q)$.

Any discrete countable group having an ergodic, free, p.m.p. and stable action is inner amenable (see [15, Proposition 4.1]). Theorem 1.1 therefore implies that any Baumslag-Solitar group is inner amenable. The latter was proved by Stalder [22]. We should compare Theorem 1.1 with Fima's result in [7] that the von Neumann algebra of $\operatorname{BS}(p, q)$ with $2 \leq|p|<|q|$ is prime, is not solid and has no Cartan subalgebra. After posting the first draft of this paper on the arXiv, we were informed by Narutaka Ozawa that the von Neumann algebra of $\operatorname{BS}(p, q)$ has property Gamma. This also implies that $\mathrm{BS}(p, q)$ is inner amenable.

Let $\mathbb{Q}$ denote the field of rational numbers. For a set of prime numbers, $S$, we define $\mathbb{Z}_{S}$ as the subring of $\mathbb{Q}$ generated by all $1 / s$ with $s \in S$. As an application of Theorem 1.1, we obtain the following:

Theorem 1.2. Let $p$ and $q$ be integers with $2 \leq|p|<|q|$. Let $S$ be a set of prime numbers dividing neither $p$ nor $q$. We denote by $\alpha$ the isomorphism from $p \mathbb{Z}_{S}$ onto $q \mathbb{Z}_{S}$ multiplying by $q / p$. Then the $H N N$ extension of $\mathbb{Z}_{S}$ relative to $\alpha$ is measure equivalent to $\mathrm{BS}(p, q)$.

In [15, Problem 4.2], Jones-Schmidt asked whether any inner amenable group has an ergodic, free, p.m.p. and stable action. Recently, Vaes [23] discovered an inner amenable group $G$ whose von Neumann algebra $L G$ is a factor and does not have property Gamma. This solved a longstanding problem posed by Effros [5]. In particular, $L G$ is not isomorphic to its tensor product with the hyperfinite $\mathrm{II}_{1}$ factor. The Vaes group $G$ could therefore be a candidate of a counterexample to the above Jones-Schmidt's question, whereas we show the following:

Theorem 1.3. The Vaes group $G$ has an ergodic, free, p.m.p. and stable action. In particular, for any amenable, discrete and countable group $A$, the group $G$ is measure equivalent to the direct product $A \times G$.

The construction of a stable action of the group $G$ is fairly similar to that for Baumslag-Solitar groups. 
This paper is organized as follows. In Section 2, we review the characterization of stable actions due to Jones-Schmidt, and introduce the notation and terminology employed throughout the paper. Theorem 1.1 is proved through Sections 3 and 4. In Section 3, focusing on a certain solvable quotient of $\mathrm{BS}(p, q)$, we construct its p.m.p. action based on the odometer action of $\mathbb{Z}$. In Section 4, using this action, we obtain an ergodic, free, p.m.p. and stable action of $\mathrm{BS}(p, q)$. As a by-product, we also find such a stable action of the normal subgroup of $\operatorname{BS}(p, q)$ generated by $a$. In the case of $p=1$, although $\mathrm{BS}(1, q)$ is amenable, our construction of stable actions is still available, and reduces to the construction in Section 3. This will help us to get an intuition for a general case. In Sections 5 and 6, Theorems 1.2 and 1.3 are proved, respectively.

\section{Preliminaries}

Let $\mathbb{N}$ denote the set of non-negative integers. Let $\mathbb{Z}_{-}$and $\mathbb{Z}_{+}$denote the set of negative integers and the set of positive integers, respectively.

2.1. Jones-Schmidt's characterization of stability. We mean by a standard probability space a standard Borel space equipped with a probability measure. All relations among Borel sets and maps that appear in the paper are understood to hold up to sets of measure zero, unless otherwise stated.

Let $(X, \mu)$ be a standard probability space. We denote by $\mathcal{B}_{X}$ the $\sigma$-algebra of Borel subsets of $X$. Let $\mathcal{R}$ be an ergodic, discrete measured equivalence relation on $(X, \mu)$ of type $\mathrm{II}_{1}$. For $x \in X$, let $\mathcal{R} x$ denote the equivalence class of $\mathcal{R}$ containing $x$. We define $[\mathcal{R}]$ as the full group of $\mathcal{R}$, that is, the group of Borel automorphisms $U$ of $(X, \mu)$ with $U x \in \mathcal{R} x$ for a.e. $x \in X$.

A sequence $\left\{B_{j}\right\}_{j \in \mathbb{N}}$ in $\mathcal{B}_{X}$ is called an asymptotically invariant (a.i.) sequence for $\mathcal{R}$ if for any $U \in[\mathcal{R}]$, we have

$$
\lim _{j} \mu\left(U B_{j} \triangle B_{j}\right)=0 .
$$

An a.i. sequence $\left\{B_{j}\right\}_{j \in \mathbb{N}}$ for $\mathcal{R}$ is called trivial if we have

$$
\lim _{j} \mu\left(B_{j}\right)\left(1-\mu\left(B_{j}\right)\right)=0 .
$$


A sequence $\left\{U_{j}\right\}_{j \in \mathbb{N}}$ in $[\mathcal{R}]$ is called an asymptotically central (a.c.) sequence for $\mathcal{R}$ if the following two conditions hold:

(a) for any $B \in \mathcal{B}_{X}$, we have $\lim _{j} \mu\left(U_{j} B \triangle B\right)=0$;

(b) for any $V \in[\mathcal{R}]$, we have $\lim _{j} \mu\left(\left\{x \in X \mid U_{j} V x \neq V U_{j} x\right\}\right)=0$.

An a.c. sequence $\left\{U_{j}\right\}_{j \in \mathbb{N}}$ for $[\mathcal{R}]$ is called trivial if we have

$$
\lim _{j} \mu\left(U_{j} B_{j} \triangle B_{j}\right)=0
$$

for any a.i. sequence $\left\{B_{j}\right\}_{j \in \mathbb{N}}$ for $\mathcal{R}$.

We mean by a discrete group a discrete and countable group. Let $G$ be a discrete group, and let $G \curvearrowright(X, \mu)$ be an ergodic p.m.p. action. We define $\mathcal{R}(G \curvearrowright(X, \mu))$ as the discrete measured equivalence relation associated with the action $G \curvearrowright(X, \mu)$, that is,

$$
\mathcal{R}(G \curvearrowright(X, \mu))=\{(g x, x) \in X \times X \mid g \in G, x \in X\},
$$

which is often denoted by $\mathcal{R}(G \curvearrowright X)$ if $\mu$ is understood from the context. An a.i. sequence for $\mathcal{R}(G \curvearrowright X)$ is also called an asymptotically invariant (a.i.) sequence for the action $G \curvearrowright(X, \mu)$. Similarly, an a.c. sequence for $\mathcal{R}(G \curvearrowright X)$ is also called an asymptotically central (a.c.) sequence for the action $G \curvearrowright(X, \mu)$. We note that a sequence $\left\{B_{j}\right\}_{j \in \mathbb{N}}$ in $\mathcal{B}_{X}$ is a.i. for the action $G \curvearrowright(X, \mu)$ if and only if for any $g \in G$, we have $\lim _{j} \mu\left(g B_{j} \triangle B_{j}\right)=0$. We also note that a sequence $\left\{U_{j}\right\}_{j \in \mathbb{N}}$ in $[\mathcal{R}(G \curvearrowright X)]$ is a.c. for the action $G \curvearrowright(X, \mu)$ if and only if it satisfies condition (a) in the definition of an a.c. sequence and the following condition:

(c) for any $g \in G$, we have $\lim _{j} \mu\left(\left\{x \in X \mid U_{j} g x \neq g U_{j} x\right\}\right)=0$.

These remarks are noticed in [15, Section 2] and [15, Remark 3.3], respectively.

Lemma 2.1. Let $\mathcal{R}$ and $\mathcal{S}$ be ergodic, discrete measured equivalence relations of type $\mathrm{II}_{1}$, on standard probability spaces $(X, \mu)$ and $(Y, v)$, respectively. Let $\pi: X \rightarrow Y$ be a Borel map with $\pi_{*} \mu=v$ and $\pi(\mathcal{R} x) \subset \mathcal{S} \pi(x)$ for a.e. $x \in X$. If $\left\{B_{j}\right\}_{j \in \mathbb{N}}$ is an a.i. sequence for $\mathcal{S}$, then $\left\{\pi^{-1}\left(B_{j}\right)\right\}_{j \in \mathbb{N}}$ is an a.i. sequence for $\mathcal{R}$.

Proof. For $j \in \mathbb{N}$, we set $A_{j}=\pi^{-1}\left(B_{j}\right)$. Pick $g \in[\mathcal{R}]$ and $\varepsilon>0$. It is enough to show that $\mu\left(g A_{j} \backslash A_{j}\right)<\varepsilon$ for any sufficiently large $j \in \mathbb{N}$. By [6, Theorem 1], we can find a discrete group $H$ and a p.m.p. action of $H$ on $(Y, v)$ such that the associated equivalence relation is equal to $\mathcal{S}$. Let $\left\{h_{n}\right\}_{n \in N}$ be an enumeration of all elements of $H$ with $N$ a countable set. There exists a countable Borel 
partition $X=\bigsqcup_{n \in N} X_{n}$ such that for any $n \in N$ and a.e. $x \in X_{n}$, we have $\pi(g x)=h_{n} \pi(x)$. Let $F$ be a finite subset of $N$ with $\mu\left(X \backslash \bigcup_{n \in F} X_{n}\right)<\varepsilon / 2$. Choose a number $J \in \mathbb{N}$ such that for any $n \in F$ and any $j \in \mathbb{N}$ with $j \geq J$, we have $v\left(h_{n} B_{j} \triangle B_{j}\right)<\varepsilon /(2|F|)$. For any $n \in N$ and $j \in \mathbb{N}$, the inclusion

$$
\begin{aligned}
g\left(A_{j} \cap X_{n}\right) \backslash A_{j} & \subset\left\{x \in X \mid \pi\left(g^{-1} x\right) \in B_{j}, h_{n} \pi\left(g^{-1} x\right)=\pi(x) \notin B_{j}\right\} \\
& \subset g \pi^{-1}\left(B_{j} \backslash h_{n}^{-1} B_{j}\right)
\end{aligned}
$$

holds. For any $j \in \mathbb{N}$ with $j \geq J$, we therefore obtain the inequality

$$
\mu\left(g A_{j} \backslash A_{j}\right) \leq \mu\left(X \backslash \bigcup_{n \in F} X_{n}\right)+\sum_{n \in F} \mu\left(g\left(A_{j} \cap X_{n}\right) \backslash A_{j}\right)<\varepsilon .
$$

The lemma is proved.

Let $\mathcal{R}_{0}$ be the ergodic hyperfinite equivalence relation on a standard probability space $\left(X_{0}, \mu_{0}\right)$ of type $\mathrm{II}_{1}$. An ergodic, discrete measured equivalence relation $\mathcal{R}$ on a standard probability space $(X, \mu)$ is called stable if $\mathcal{R}$ is isomorphic to the direct product $\mathcal{R} \times \mathcal{R}_{0}$ on $\left(X \times X_{0}, \mu \times \mu_{0}\right)$ defined as

$$
\mathcal{R} \times \mathcal{R}_{0}=\left\{\left(\left(x, x_{0}\right),\left(y, y_{0}\right)\right) \in\left(X \times X_{0}\right)^{2} \mid(x, y) \in \mathcal{R},\left(x_{0}, y_{0}\right) \in \mathcal{R}_{0}\right\} .
$$

An ergodic p.m.p. action $G \curvearrowright(X, \mu)$ is called stable if $\mathcal{R}(G \curvearrowright X)$ is stable. Jones-Schmidt obtained the following characterization of stability.

Theorem 2.2 ([15, Theorem 3.4]). An ergodic discrete measured equivalence relation of type $\mathrm{II}_{1}$ is stable if and only if it has a non-trivial a.c. sequence.

2.2. Abelian groups associated with integers. Fix an integer $l$ with $l \geq 2$. Put $E=\mathbb{Z}$. We define a ring $E_{l}$ as the projective limit $\lim E / l^{n} E$, which is compact and unital. We have an embedding of $E$ into $E_{l}$ through the canonical projection from $E$ onto $E / l^{n} E$. Each element $x$ of $E_{l}$ is uniquely written as the formal sum $x=\sum_{i=0}^{\infty} x_{i} l^{i}$ with $x_{i} \in\{0,1, \ldots, l-1\}$ for any $i \in \mathbb{N}$.

The additive group $E_{l}$ is torsion-free. In fact, if $k$ is a positive integer and $a$ is an element of $E_{l}$ with $k a=0$, then choosing a sequence $\left\{m_{i}\right\}_{i \in \mathbb{N}}$ in $E$ approaching $a$, we obtain the sequence $\left\{k m_{i}\right\}_{i \in \mathbb{N}}$ in $E$ approaching 0 . It follows that for any $n \in \mathbb{N}$, there exists a number $I \in \mathbb{N}$ such that for any $i \in \mathbb{N}$ with $i \geq I$, the number $k m_{i}$ is divisible by $l^{n}$. This is equivalent to that for any $n \in \mathbb{N}$, there exists a number $J \in \mathbb{N}$ such that for any $i \in \mathbb{N}$ with $i \geq J$, the number $m_{i}$ is divisible by $l^{n}$. We therefore have $a=0$. The claim is proved. 
Let $R_{l}$ denote the ring of fractions of $E_{l}$ by the multiplicative subset $S=$ $\left\{l^{n} \in E \mid n \in \mathbb{N}\right\}$. The ring $R_{l}$ consists of equivalence classes of all elements in $E_{l} \times S$, where two elements $(a, s),(b, t) \in E_{l} \times S$ are equivalent if and only if there exists an element $u \in S$ with $(a t-b s) u=0$. The equivalence class of $(a, s) \in E_{l} \times S$ is denoted by $a / s$. Using that the additive group $E_{l}$ is torsion-free, we can show that $E_{l}$ is naturally a subring of $R_{l}$. The subring of $R_{l}$ generated by $1 / l$ is isomorphic to the ring $\mathbb{Z}[1 / l]$.

Suppose that we have two coprime integers $p, q$ with $2 \leq p<q$ and $l=p q$. We define $R_{l, q}$ as the subgroup of $R_{l}$ generated by $E_{l}$ and all elements of the form $1 / q^{n}$ with $n \in \mathbb{N}$. Similarly, we define $R_{l, p}$ as the subgroup of $R_{l}$ generated by $E_{l}$ and all elements of the form $1 / p^{m}$ with $m \in \mathbb{N}$. Each element $x$ of $R_{l, q} \backslash E_{l}$ is uniquely written as the formal sum

$$
x=x_{n} q^{n}+x_{n+1} q^{n+1}+\cdots+x_{-1} q^{-1}+\sum_{j=0}^{\infty} x_{j} l^{j}
$$

with $n \in \mathbb{Z}_{-} ; x_{i} \in\{0,1, \ldots, q-1\}$ for any integer $i$ with $n \leq i \leq-1 ; x_{n} \neq 0$; and $x_{j} \in\{0,1, \ldots, l-1\}$ for any $j \in \mathbb{N}$. Since $p$ and $q$ are coprime, the element $x$ is also uniquely written as the formal sum

$$
x=y_{n}\left(\frac{q}{p}\right)^{n}+y_{n+1}\left(\frac{q}{p}\right)^{n+1}+\cdots+y_{-1}\left(\frac{q}{p}\right)^{-1}+\sum_{j=0}^{\infty} y_{j} l^{j}
$$

with $y_{i} \in\{0,1, \ldots, q-1\}$ for any integer $i$ with $n \leq i \leq-1 ; y_{n} \neq 0$; and $y_{j} \in\{0,1, \ldots, l-1\}$ for any $j \in \mathbb{N}$. The coefficients $y_{n}, y_{n+1}, \ldots$ are inductively determined by $x_{n}, x_{n+1}, \ldots$, and the difference $\sum_{j=0}^{\infty} x_{j} l^{j}-\sum_{j=0}^{\infty} y_{j} l^{j}$ lies in $E$. We also have similar expansions of elements of $R_{l, p} \backslash E_{l}$.

\section{Actions of certain solvable quotients}

Throughout this section, we fix two coprime integers $p, q$ with $1 \leq p<q$. We set $G_{0}=G_{0}(p, q)=\mathbb{Z}[1 / p, 1 / q]$. Let $a$ denote the multiplicative unit 1 in $G_{0}$. Let $t$ denote the automorphism of the group $G_{0}$ multiplying by $q / p$. We define a discrete group $G=G(p, q)$ as the semi-direct product of $G_{0}$ and the infinite cyclic group generated by $t$. Note that for any positive integer $r$, the group $G$ is a quotient of the group $\mathrm{BS}(r p, r q)$. The aim of this section is to construct an interesting ergodic p.m.p. action $G \curvearrowright(Y, v)$, which will be used in the next section. 
3.1. The case of $\boldsymbol{p}=\mathbf{1}$. We assume $p=1$. The group $G$ then has the presentation $\left\langle a, t \mid t a t^{-1}=a^{q}\right\rangle$. We set

$$
Y=\prod_{\mathbb{Z}}\{0,1, \ldots, q-1\}
$$

and define a probability measure $v$ on $Y$ as the direct product of the uniformly distributed probability measure on $\{0,1, \ldots, q-1\}$. Let $a$ act on $Y$ by the odometer adding 1 to the 0th coordinate and increasing digits toward positive coordinates. More precisely, for each $y=\left(y_{n}\right)_{n \in \mathbb{Z}} \in Y$, the element $a y=\left(z_{n}\right)_{n \in \mathbb{Z}}$ is determined by the formula $z_{n}=y_{n}$ for $n \in \mathbb{Z}_{-}$and the equality

$$
1+\sum_{n=0}^{\infty} y_{n} q^{n}=\sum_{n=0}^{\infty} z_{n} q^{n}
$$

in the group $E_{q}$. Let $t$ act on $Y$ by the shift toward the right. Namely, for each $y=\left(y_{n}\right)_{n \in \mathbb{Z}} \in Y$, the element $t y=\left(w_{n}\right)_{n \in \mathbb{Z}}$ is determined by the formula $w_{n}=y_{n-1}$ for $n \in \mathbb{Z}$. This defines an ergodic p.m.p. action of $G$ on $(Y, v)$.

3.2. The case of $p>1$. We assume $p>1$. The group $G$ then has the presentation so that generators are $a_{i}$ for $i \in \mathbb{Z}$ and $t$, and relations are $\left[a_{i}, a_{j}\right]=e$, $a_{i+1}^{p}=a_{i}^{q}$ and $t a_{i} t^{-1}=a_{i+1}$ for any $i, j \in \mathbb{Z}$. Note that for each $i \in \mathbb{Z}, a_{i}$ corresponds to the number $(q / p)^{i} \in \mathbb{Z}[1 / p, 1 / q]$. Under this identification, we have $a_{0}=a$.

We set

$$
\begin{aligned}
Y_{-} & =\prod_{\mathbb{Z}_{-}}\{0,1, \ldots, q-1\}, \\
Y_{0} & =\prod_{\mathbb{N}}\{0,1, \ldots, p q-1\}, \\
Y_{+} & =\prod_{\mathbb{Z}_{+}}\{0,1, \ldots, p-1\} .
\end{aligned}
$$

We define a probability measure $\nu_{-}$on $Y_{-}$as the direct product of the uniformly distributed probability measure on $\{0,1, \ldots, q-1\}$. Similarly, we define probability measures $v_{0}$ and $v_{+}$on $Y_{0}$ and $Y_{+}$, respectively. We set

$$
(Y, v)=\left(Y_{-}, v_{-}\right) \times\left(Y_{0}, v_{0}\right) \times\left(Y_{+}, v_{+}\right) .
$$

The set $Y_{0}$ is identified with the group $E_{p q}$ under the map sending each element $\left(x_{k}\right)_{k \in \mathbb{N}}$ of $Y_{0}$ to the sum $\sum_{k \in \mathbb{N}} x_{k}(p q)^{k}$. In the following argument, it is convenient to regard each element of $Y$,

$$
y=\left(\left(y_{n}\right)_{n \in \mathbb{Z}_{-}}, y_{0},\left(y_{m}\right)_{m \in \mathbb{Z}_{+}}\right) \in Y_{-} \times Y_{0} \times Y_{+},
$$


as the formal sum

$$
\cdots+y_{-2}\left(\frac{q}{p}\right)^{-2}+y_{-1}\left(\frac{q}{p}\right)^{-1}+y_{0}+y_{1} \frac{q}{p}+y_{2}\left(\frac{q}{p}\right)^{2}+\cdots .
$$

We now define elements $a_{i} y \in Y$ for $i \in \mathbb{Z}$ and $t y \in Y$.

Let $a_{0}$ act on $Y$ by adding 1 on $Y_{0}$, that is, set

$$
a_{0} y=\left(\left(y_{n}\right)_{n \in \mathbb{Z}_{-}}, 1+y_{0},\left(y_{m}\right)_{m \in \mathbb{Z}_{+}}\right),
$$

where $Y_{0}$ is identified with the group $E_{p q}$. For each $i \in \mathbb{Z}_{-}$, let $a_{i}$ act on $Y$ by adding $(q / p)^{i}$ to $y$. More precisely, the element

$$
a_{i} y=\left(\left(z_{n}\right)_{n \in \mathbb{Z}_{-}}, z_{0},\left(z_{m}\right)_{m \in \mathbb{Z}_{+}}\right) \in Y_{-} \times Y_{0} \times Y_{+}
$$

of $Y$ is determined by the formula $z_{n}=y_{n}$ for any $n \in \mathbb{Z}$ with $n \leq i-1$ or $n \geq 1$ and the equation

$$
\left(\frac{q}{p}\right)^{i}+\sum_{n=i}^{-1} y_{n}\left(\frac{q}{p}\right)^{n}+y_{0}=\sum_{n=i}^{-1} z_{n}\left(\frac{q}{p}\right)^{n}+z_{0}
$$

in the group $R_{p q}$. Similarly, for each $j \in \mathbb{Z}_{+}$, let $a_{j}$ act on $Y$ by adding $(q / p)^{j}$ to $y$. More precisely, the element

$$
a_{j} y=\left(\left(w_{n}\right)_{n \in \mathbb{Z}_{-}}, w_{0},\left(w_{m}\right)_{m \in \mathbb{Z}_{+}}\right) \in Y_{-} \times Y_{0} \times Y_{+}
$$

of $Y$ is determined by the formula $w_{n}=y_{n}$ for any $n \in \mathbb{Z}$ with $n \leq-1$ or $n \geq j+1$ and the equation

$$
y_{0}+\sum_{m=1}^{j} y_{m}\left(\frac{q}{p}\right)^{m}+\left(\frac{q}{p}\right)^{j}=w_{0}+\sum_{m=1}^{j} w_{m}\left(\frac{q}{p}\right)^{m}
$$

in the group $R_{p q}$. One can check that for any $i \in \mathbb{Z}$, the Borel automorphism $a_{i}$ of $Y$ defined above preserves the measure $v$, and that for any $i, j \in \mathbb{Z}$, the relations $\left[a_{i}, a_{j}\right]=e$ and $a_{i+1}^{p}=a_{i}^{q}$ hold. 
Let $\eta: Y_{0} \rightarrow\{0,1, \ldots, p-1\}$ be the Borel map defined so that for any $x \in E_{p q}$, the element $x-\eta(x)$ belongs to $p E_{p q}$. We define $t y \in Y$ by the formula

$$
t y=\left(\left(y_{n-1}\right)_{n \in \mathbb{Z}_{-}}, y_{-1}+\frac{q}{p}\left(y_{0}-\eta\left(y_{0}\right)\right),\left(\eta\left(y_{0}\right), y_{1}, y_{2}, \ldots\right)\right) .
$$

Lemma 3.1. The map $t: Y \rightarrow Y$ defined above is a Borel automorphism of $Y$ and preserves the measure $v$.

Proof. Let $\zeta: Y_{0} \rightarrow\{0,1, \ldots, q-1\}$ be the Borel map defined so that for any $x \in E_{p q}$, the element $x-\zeta(x)$ belongs to $q E_{p q}$. The inverse of the map $t$ is the map sending each element $y$ of $Y$, written as

$$
y=\left(\left(y_{n}\right)_{n \in \mathbb{Z}_{-}}, y_{0},\left(y_{m}\right)_{m \in \mathbb{Z}_{+}}\right) \in Y_{-} \times Y_{0} \times Y_{+},
$$

to

$$
\left(\left(\ldots, y_{-2}, y_{-1}, \zeta\left(y_{0}\right)\right), \frac{p}{q}\left(y_{0}-\zeta\left(y_{0}\right)\right)+y_{1},\left(y_{m+1}\right)_{m \in \mathbb{Z}_{+}}\right) \in Y_{-} \times Y_{0} \times Y_{+} .
$$

The map $t$ is thus a Borel automorphism of $Y$.

Let $N$ and $M$ be positive integers. Pick

$$
k_{-N}, k_{-N+1}, \ldots, k_{-1} \in\{0,1, \ldots, q-1\}
$$

and

$$
l_{1}, l_{2}, \ldots, l_{M} \in\{0,1, \ldots, p-1\} .
$$

We also pick $i \in\{0,1, \ldots, p-1\}$ and a Borel subset $A$ of $i+p E_{p q}$. We define the Borel subset of $Y$,

$$
\begin{array}{r}
B=\left\{\left(\left(y_{n}\right)_{n}, y_{0},\left(y_{m}\right)_{m}\right) \in Y \mid y_{n}=k_{n}, \text { for all } n \in\{-N,-N+1, \ldots,-1\},\right. \\
\left.y_{0} \in A, y_{m}=l_{m}, \text { for all } m \in\{1,2, \ldots, M\}\right\} .
\end{array}
$$

We then have

$$
v(B)=q^{-N} v_{0}(A) p^{-M}
$$

and

$$
\begin{aligned}
& t B=\left\{\left(\left(y_{n}\right)_{n}, y_{0},\left(y_{m}\right)_{m}\right) \in Y \mid\right. \\
& y_{n}=k_{n-1}, \text { for all } n \in\{-N+1,-N+2, \ldots,-1\}, \\
& y_{0} \in k_{-1}+\frac{q}{p}(-i+A), y_{1}=i, y_{m}=l_{m-1}, \\
& \quad \text { for all } m \in\{2,3, \ldots, M+1\}\} .
\end{aligned}
$$


The equality

$$
v(t B)=q^{-N+1} \frac{p}{q} v_{0}(A) p^{-1} p^{-M}=v(B)
$$

thus holds. It follows that $t$ preserves $v$.

One can directly check the relation $t a_{i} t^{-1}=a_{i+1}$ as Borel automorphisms of $Y$ for any $i \in \mathbb{Z}$. We therefore obtain a p.m.p. action of $G$ on $(Y, v)$.

Let $\mathcal{S}_{0}$ denote the equivalence relation on $(Y, v)$ defined as follows: Two elements of $Y$,

$$
y=\left(\left(y_{n}\right)_{n \in \mathbb{Z}_{-}},\left(\bar{y}_{i}\right)_{i \in \mathbb{N}},\left(y_{m}\right)_{m \in \mathbb{Z}_{+}}\right)
$$

and

$$
z=\left(\left(z_{n}\right)_{n \in \mathbb{Z}_{-}},\left(\bar{z}_{i}\right)_{i \in \mathbb{N}},\left(z_{m}\right)_{m \in \mathbb{Z}_{+}}\right),
$$

are equivalent in $\mathcal{S}_{0}$ if and only if

$$
\begin{array}{cl}
y_{n}=z_{n} & \text { for all but finitely many } n \in \mathbb{Z}_{-}, \\
\bar{y}_{i}=\bar{z}_{i} & \text { for all but finitely many } i \in \mathbb{N}, \text { and } \\
y_{m}=z_{m} & \text { for all but finitely many } m \in \mathbb{Z}_{+} .
\end{array}
$$

For a.e. $y \in Y$, the equivalence class of $y$ with respect to $\mathcal{S}_{0}$ is equal to the orbit of $y$ under the action of the subgroup $G_{0}$ on $Y$. The action of $G_{0}$ on $(Y, v)$ is therefore ergodic.

Remark 3.2. In the above construction of the action $G \curvearrowright(Y, v)$, if we substitute 1 for $p$, then $Y_{+}$consists of a single point, and the action coincides with the action constructed in Section 3.1. We however decided to discuss the cases of $p=1$ and $p>1$ individually to avoid confusion.

Let $\theta: Y \rightarrow Y_{0}$ denote the projection. The following lemma will be used in the proof of Lemma 4.5.

Lemma 3.3. For any $s \in G_{0}$ and a.e. $y \in Y$, there exists a number $K=K(s, y) \in$ $\mathbb{N}$ such that for any $k \in \mathbb{N}$ with $k \geq K$,

$$
\theta\left(t^{-k} s y\right)=\theta\left(t^{-k} y\right) .
$$

Proof. The product of two elements of the group $G_{0}$ is denoted multiplicatively. Suppose that the lemma is true for $s_{1}, s_{2} \in G_{0}$. For a.e. $y \in Y$ and any integer $k$ bigger than $K\left(s_{1}, s_{2} y\right)$ and $K\left(s_{2}, y\right)$, the equality

$$
\theta\left(t^{-k}\left(s_{1} s_{2}\right) y\right)=\theta\left(t^{-k} s_{1}\left(s_{2} y\right)\right)=\theta\left(t^{-k} s_{2} y\right)=\theta\left(t^{-k} y\right)
$$

holds. The lemma is therefore true for $s_{1} s_{2}$. 
It is enough to show the lemma when $s=t^{i} a t^{-i}=(q / p)^{i}$ for some $i \in \mathbb{Z}$ because all elements of this form generate $G_{0}$. If $i$ is non-negative, then for any integer $k$ and any $y \in Y$, we have the equalities

$$
t^{-k} s y=t^{-k+i+1}\left(t^{-i-1} s t^{i+1}\right)\left(t^{-i-1} y\right)
$$

and

$$
t^{-i-1} s t^{i+1}=\left(\frac{q}{p}\right)^{-1} .
$$

It is therefore enough to show the lemma when $s=(q / p)^{i}$ with $i \in \mathbb{Z}_{-}$.

For $n \in \mathbb{Z}_{-}$, let $\rho_{n}: Y \rightarrow\{0,1, \ldots, q-1\}$ denote the projection onto the $n$th coordinate of $Y_{-}$. For $m \in \mathbb{N}$, we define a Borel map

$$
\tau_{m}: Y \longrightarrow\{0,1, \ldots, q-1\}
$$

by

$$
\tau_{m}(y)=\rho_{-1}\left(t^{-m-1} y\right) \quad \text { for } y \in Y .
$$

We have the equality $\rho_{n}\left(t^{-m+n} y\right)=\rho_{-1}\left(t^{-m-1} y\right)$ for any $m \in \mathbb{N}, n \in \mathbb{Z}_{-}$and $y \in Y$.

Claim 3.4. The Borel subset of $Y$,

$$
A=\left\{y \in Y \mid \tau_{m}(y) \geq q-p, \text { for all } m \in \mathbb{N}\right\},
$$

has zero v-measure.

Proof. For $k \in \mathbb{N}$, we set

$$
A_{k}=\left\{y \in Y \mid \tau_{m}(y) \geq q-p, \text { for all } m \in\{0,1, \ldots, k\}\right\} .
$$

For any $k \in \mathbb{N}$ and any $y=\left(\left(y_{n}\right)_{n}, y_{0},\left(y_{m}\right)_{m}\right) \in Y$, we have

$$
t^{-k-1} y=\cdots+y_{-1}\left(\frac{q}{p}\right)^{-k-2}+\sum_{n=-k-1}^{-1} \tau_{n+k+1}(y)\left(\frac{q}{p}\right)^{n}+\cdots .
$$

The inclusion

$$
t^{-k-1} A_{k} \subset \bigcap_{n=-k-1}^{-1} \rho_{n}^{-1}(\{q-p, q-p+1, \ldots, q-1\})
$$

thus holds. It follows that $v\left(A_{k}\right)=v\left(t^{-k-1} A_{k}\right) \leq(p / q)^{k+1}$. The inclusion $A \subset A_{k}$ for any $k \in \mathbb{N}$ implies that $v(A)=0$. 
Claim 3.5. Fix $n, m \in \mathbb{Z}_{+}$. Pick a number $z_{l} \in\{0,1, \ldots, q-1\}$ indexed by each integer $l$ with $-n-m+1 \leq l \leq-n$. If $z_{-n} \leq q-p-1$, then for each integer $l$ with $-n-m+1 \leq l \leq-n$, there exists a unique number $z_{l}^{\prime} \in\{0,1, \ldots, q-1\}$ with

$$
q\left(\frac{q}{p}\right)^{-n-m}+\sum_{l=-n-m+1}^{-n} z_{l}\left(\frac{q}{p}\right)^{l}=\sum_{l=-n-m+1}^{-n} z_{l}^{\prime}\left(\frac{q}{p}\right)^{l} .
$$

Proof. We find the number $z_{l}^{\prime}$ by induction on $m$. If $m=1$, then the equation

$$
q\left(\frac{q}{p}\right)^{-n-1}+z_{-n}\left(\frac{q}{p}\right)^{-n}=\left(p+z_{-n}\right)\left(\frac{q}{p}\right)^{-n}
$$

holds. It thus suffices to set $z_{-n}^{\prime}=p+z_{-n}$. In general, if $p+z_{-n-m+1} \leq q-1$, then we set $z_{-n-m+1}^{\prime}=p+z_{-n-m+1}$ and $z_{l}^{\prime}=z_{l}$ for any $l$ with $-n-m+2 \leq l \leq-n$. Otherwise, the left hand side of the equation in the claim is equal to

$$
\left(p+z_{-n-m+1}-q\right)\left(\frac{q}{p}\right)^{-n-m+1}+q\left(\frac{q}{p}\right)^{-n-m+1}+\sum_{l=-n-m+2}^{-n} z_{l}\left(\frac{q}{p}\right)^{l} .
$$

By using the inequality $0 \leq p+z_{-n-m+1}-q \leq q-1$ and the hypothesis of the induction, we can find the number $z_{l}^{\prime}$ in the claim. Uniqueness of $z_{l}^{\prime}$ holds because $p$ and $q$ are coprime.

We now prove Lemma 3.3 for $s=(q / p)^{i} \in G_{0}$ with $i \in \mathbb{Z}_{-}$. Fix $K \in \mathbb{N}$. For any $k \in \mathbb{N}$ with $k>K$ and any $y=\left(\left(y_{n}\right)_{n}, y_{0},\left(y_{m}\right)_{m}\right) \in Y$, we have

$$
t^{-k} y=\cdots+\sum_{n=i-k}^{-1-k} y_{n+k}\left(\frac{q}{p}\right)^{n}+\sum_{m=-k}^{-k+K} \tau_{m+k}(y)\left(\frac{q}{p}\right)^{m}+\cdots .
$$

The equalities $t^{-k} s y=t^{-k} s t^{k}\left(t^{-k} y\right)$ and $t^{-k} s t^{k}=(q / p)^{i-k}$ imply that $t^{-k} s y$ is obtained by adding $(q / p)^{i-k}$ to $t^{-k} y$. If $y_{i}<q-1$, then the coordinates of $t^{-k} s y$ and $t^{-k} y$ except for the $(i-k)$ th one of $Y_{-}$are equal. If $y_{i}=q-1$ and $\tau_{K}(y) \leq q-p-1$, then by Claim 3.5, the coordinates of $t^{-k} s y$ and $t^{-k} y$ except for the $j$ th one of $Y_{-}$with $i-k \leq j \leq-k+K$ are equal. We thus have $\theta\left(t^{-k} s y\right)=\theta\left(t^{-k} y\right)$ for any $k \in \mathbb{N}$ with $k>K$ and any $y \in Y$ with $\tau_{K}(y) \leq q-p-1$. Lemma 3.3 then follows from Claim 3.4. 


\section{Stable actions of Baumslag-Solitar groups}

Throughout this section, we fix two coprime integers $p, q$ with $1 \leq p<q$, and fix a positive integer $r$ with $r p \geq 2$. We set

$$
\Gamma=\mathrm{BS}(r p, r q)=\left\langle a, t \mid t a^{r p} t^{-1}=a^{r q}\right\rangle
$$

and set $E=\langle a\rangle$. Let $H$ be the normal subgroup of $\Gamma$ generated by $a$. The quotient group $\Gamma / H$ is isomorphic to $\mathbb{Z}$ and is generated by the image of $t$. Let $G_{0}=$ $G_{0}(p, q)$ and $G=G(p, q)$ be the groups defined in the beginning of Section 3. We have the surjective homomorphism $\epsilon: \Gamma \rightarrow G$ sending $a$ to the multiplicative unit 1 of $G_{0}$ and sending $t$ to the automorphism of $G_{0}$ multiplying by $q / p$. Let $N$ be the kernel of $\epsilon$.

We set

$$
X=\prod_{\mathbb{N}}\{0,1\}
$$

and define a probability measure $\mu$ on $X$ as the direct product of the uniformly distributed probability measure on $\{0,1\}$. We define an ergodic p.m.p. action of $\Gamma$ on $(X, \mu)$ so that $a$ acts on it trivially, and $t$ acts on it by the odometer adding 1 to the 0th coordinate of $X$ and increasing digits toward the right. Note that $H$ acts on $X$ trivially. For $j \in \mathbb{N}$ and $l_{0}, l_{1}, \ldots, l_{j} \in\{0,1\}$, we set

$$
X\left(l_{0}, l_{1}, \ldots, l_{j}\right)=\left\{\left(x_{n}\right)_{n \in \mathbb{N}} \in X \mid x_{0}=l_{0}, x_{1}=l_{1}, \ldots, x_{j}=l_{j}\right\} .
$$

Let $G \curvearrowright(Y, v)$ be the action constructed in Section 3. Let $\Gamma$ act on $(Y, v)$ through the homomorphism $\epsilon: \Gamma \rightarrow G$.

\subsection{Co-induced actions. We set}

$$
Z_{0}=\prod_{\mathbb{N}}\{0,1, \ldots, p q-1\}
$$

and define a probability measure $\xi_{0}$ on $Z_{0}$ as the direct product of the uniformly distributed probability measure on $\{0,1, \ldots, p q-1\}$. Let $E$ act on $\left(Z_{0}, \xi_{0}\right)$ by odometers so that $a$ adds 1 to the 0th coordinate and increases digits toward the right. We set

$$
(Z, \xi)=\prod_{\Gamma / E}\left(Z_{0}, \xi_{0}\right)
$$

and define an action of $\Gamma$ on $(Z, \xi)$ as the action co-induced from the action $E \curvearrowright$ $\left(Z_{0}, \xi_{0}\right)$. Namely, fixing a section $s: \Gamma / E \rightarrow \Gamma$ for the canonical map from $\Gamma$ onto $\Gamma / E$, for $\gamma \in \Gamma$ and $f \in Z$, we define an element $\gamma f \in Z$ by the equation

$$
(\gamma f)(\alpha)=b^{-1} f(\beta)
$$


for $\alpha \in \Gamma / E$, where $b \in E$ and $\beta \in \Gamma / E$ are the unique elements determined by the equation $s(\beta) b=\gamma^{-1} s(\alpha)$. This indeed defines a p.m.p. action of $\Gamma$ on $(Z, \xi)$. One can check that this action is essentially free, that is, the stabilizer of a.e. point of $Z$ for the action is trivial, by using that the action of $E$ on $\left(Z_{0}, \xi_{0}\right)$ is essentially free. The construction of co-induced actions appears in [12, Section 3.4].

Lemma 4.1. The following assertions hold.

(i) For any $g \in \Gamma$, there exist $K, K^{\prime}, L, L^{\prime} \in \mathbb{N}$ such that $K+L=K^{\prime}+L^{\prime}$ and for any $k, l \in \mathbb{N}$, we have $g a^{r p^{K+k}} q^{L+l} g^{-1}=a^{r p^{K^{\prime}+k}} q^{L^{\prime}+l}$.

(ii) For any sequence $\left\{n_{k}\right\}_{k \in \mathbb{N}}$ of positive integers and any Borel subset $B$ of $Z$, we have

$$
\lim _{k \rightarrow \infty} \xi\left(a^{r n_{k}(p q)^{k}} B \triangle B\right)=0 .
$$

This convergence is indeed uniform with respect to the sequence $\left\{n_{k}\right\}_{k \in \mathbb{N}}$. Namely, for any $\varepsilon>0$ and any Borel subset $B$ of $Z$, there exists $K \in \mathbb{N}$ such that for any sequence $\left\{n_{k}\right\}_{k \in \mathbb{N}}$ of positive integers and for any $k \in \mathbb{N}$ with $k \geq K$, we have $\xi\left(a^{r n_{k}(p q)^{k}} B \triangle B\right)<\varepsilon$.

(iii) The action of $N$ on $(Z, \xi)$ is ergodic.

Proof. The presentation of $\Gamma$ implies that for any $g \in \Gamma$, there exist $K, L \in \mathbb{N}$ with $g a^{r p^{K} q^{L}} g^{-1} \in E$, which is equal to $a^{r p^{K^{\prime}}} q^{L^{\prime}}$ for some $K^{\prime}, L^{\prime} \in \mathbb{N}$ with $K+L=K^{\prime}+L^{\prime}$. The equality in assertion (i) then follows.

By the definition of odometers, for any sequence $\left\{m_{k}\right\}_{k \in \mathbb{N}}$ of positive integers and any Borel subset $C$ of $Z_{0}$, we have

$$
\lim _{k \rightarrow \infty} \xi_{0}\left(a^{m_{k}(p q)^{k}} C \triangle C\right)=0,
$$

and this convergence is uniform with respect to the sequence $\left\{m_{k}\right\}_{k \in \mathbb{N}}$. Let $F$ be a finite subset of $\Gamma / E$. By assertion (i), there exist $K, L \in \mathbb{N}$ satisfying the following: For any $\alpha \in F$, we have $K_{\alpha}, L_{\alpha} \in \mathbb{N}$ such that $K+L=K_{\alpha}+L_{\alpha}$, and if $\left\{n_{k}\right\}_{k \in \mathbb{N}}$ is a sequence of positive integers, then for any $k \in \mathbb{N}$ with $k \geq K+L$, the equality

$$
\begin{aligned}
a^{-r n_{k}(p q)^{k}} s(\alpha) & =a^{-r n_{k} p^{K} q^{L} p^{k-K} q^{k-L}} s(\alpha) \\
& =s(\alpha) a^{-r n_{k} p^{K \alpha} q^{L \alpha} p^{k-K} q^{k-L}} \\
& =s(\alpha) a^{-r n_{k} p^{K \alpha+L} q^{L \alpha+K}(p q)^{k-K-L}}
\end{aligned}
$$


holds. Let $\pi_{F}: Z \rightarrow \prod_{F} Z_{0}$ be the natural projection. For any sequence $\left\{n_{k}\right\}_{k \in \mathbb{N}}$ of positive integers and any Borel subset $D$ of $\prod_{F} Z_{0}$, we therefore have

$$
\lim _{k \rightarrow \infty} \xi\left(a^{r n_{k}(p q)^{k}} \pi_{F}^{-1}(D) \triangle \pi_{F}^{-1}(D)\right)=0
$$

uniformly with respect to the sequence $\left\{n_{k}\right\}_{k \in \mathbb{N}}$. Assertion (ii) is proved.

The assumption $r p \geq 2$ implies that $N$ is infinite. Assertion (iii) holds because $N$ acts on $\Gamma / E$ freely.

We set

$$
(W, \omega)=(X, \mu) \times(Y, v) \times(Z, \xi)
$$

and define a p.m.p. action $\Gamma \curvearrowright(W, \omega)$ as the diagonal action so that for $\gamma \in \Gamma$ and $w=(x, y, z) \in W$, we have $\gamma w=(\gamma x, \gamma y, \gamma z)$. The action $\Gamma \curvearrowright(W, \omega)$ is ergodic because so are the actions $N \curvearrowright(Z, \xi), H / N \curvearrowright(Y, \nu)$ and $\Gamma / H \curvearrowright$ $(X, \mu)$. The action $\Gamma \curvearrowright(W, \omega)$ is essentially free because so is the action $\Gamma \curvearrowright$ $(Z, \xi)$. We will show that the action $\Gamma \curvearrowright(W, \omega)$ has a non-trivial a.c. sequence and is therefore stable. This is enough to show Theorem 1.1 for $\Gamma$ because one can find a $\Gamma$-invariant conull Borel subset of $W$ on which $\Gamma$ acts freely.

4.2. The case of $p=1$. We assume $p=1$. Fix a positive integer $M$ with $q^{M}>r$. We set

$$
S=\prod_{\mathbb{N}}\left\{0,1, \ldots, q^{M}-1\right\}
$$

and define a probability measure $\sigma$ on $S$ as the direct product of the uniformly distributed probability measure on $\left\{0,1, \ldots, q^{M}-1\right\}$. The odometer relation on $(S, \sigma)$, denoted by $\mathcal{R}_{0}$, is defined so that two elements $\left(c_{i}\right)_{i},\left(c_{i}^{\prime}\right)_{i} \in S$ are equivalent if and only if for any sufficiently large $i \in \mathbb{N}$, we have $c_{i}=c_{i}^{\prime}$.

Recall that we have constructed the action of $\Gamma$ on the space

$$
Y=\prod_{\mathbb{Z}}\{0,1, \ldots, q-1\}
$$

with the probability measure $v$. Let $\Gamma$ act on $X \times Y$ diagonally. For each $j \in \mathbb{N}$, we define $\theta_{j}: X \times Y \rightarrow\{0,1, \ldots, q-1\}$ as the projection onto the $j$ th coordinate of $Y$.

We define a Borel map $\pi: X \times Y \rightarrow S$ as follows. Pick an element

$$
\lambda=\left(\left(x_{n}\right)_{n \in \mathbb{N}},\left(y_{m}\right)_{m \in \mathbb{Z}}\right) \in X \times Y .
$$

For each $j \in \mathbb{N}$, the $j$ th coordinate of $\pi(\lambda) \in S$, denoted by $\pi(\lambda)_{j}$, is defined by

$$
\pi(\lambda)_{j}=\sum_{k=0}^{M-1} \theta_{j M+k}\left(t^{-x_{0}-2 x_{1}-\cdots-2^{j} x_{j}} \lambda\right) q^{k}=\sum_{k=0}^{M-1} y_{j M+k+x_{0}+2 x_{1}+\cdots+2^{j} x_{j}} q^{k} .
$$


Lemma 4.2. In the above notation, the following assertions hold:

(i) the equality $\pi_{*}(\mu \times v)=\sigma$ holds;

(ii) for any $j \in \mathbb{N}$, the map $\pi(\cdot)_{j}: X \times Y \rightarrow\left\{0,1, \ldots, q^{M}-1\right\}$ is invariant under the restriction of $t$ to the subset of $X \times Y$,

$$
(X \backslash X(\underbrace{1, \ldots, 1}_{j+1})) \times Y
$$

that is, for any element $\lambda$ of this subset, we have $\pi(t \lambda)_{j}=\pi(\lambda)_{j}$;

(iii) for a.e. $\lambda \in X \times Y$, we have $\pi(\Gamma \lambda) \subset \mathcal{R}_{0} \pi(\lambda)$;

Proof. Pick $d \in \mathbb{N}$ and $h_{0}, h_{1}, \ldots, h_{d} \in\left\{0,1, \ldots, q^{M}-1\right\}$, and set

$$
T=\left\{\left(c_{i}\right)_{i \in \mathbb{N}} \in S \mid c_{0}=h_{0}, c_{1}=h_{1}, \ldots, c_{d}=h_{d}\right\}
$$

To prove assertion (i), it suffices to show that $(\mu \times v)\left(\pi^{-1}(T)\right)=q^{-(d+1) M}$. We set

$$
\Omega=X(\underbrace{0, \ldots, 0}_{d+1})
$$

and

$$
A_{j}=\left\{\left(y_{n}\right)_{n \in \mathbb{Z}} \in Y \mid \sum_{k=0}^{M-1} y_{j M+k} q^{k}=h_{j}\right\}
$$

for each $j \in\{0,1, \ldots, d\}$. For each integer $l$ with $0 \leq l \leq 2^{d+1}-1$, we denote by $l_{0}, l_{1}, \ldots, l_{d} \in\{0,1\}$ as the numbers determined by the equation

$$
l=l_{0}+2 l_{1}+\cdots+2^{d} l_{d}
$$

We then have the equality

$$
\pi^{-1}(T)=\bigsqcup_{l=0}^{2^{d+1}-1}\left(t^{l} \Omega\right) \times\left(\bigcap_{j=0}^{d} t^{l_{0}+2 l_{1}+\cdots+2^{j}} l_{j} A_{j}\right) .
$$

Fix an integer $l$ with $0 \leq l \leq 2^{d+1}-1$. For any $j \in\{0,1, \ldots, d\}$, we have

$$
t^{l_{0}+2 l_{1}+\cdots+2^{j} l_{j}} A_{j}=\left\{\left(y_{n}\right)_{n \in \mathbb{Z}} \in Y \mid \sum_{k=0}^{M-1} y_{j M+k+l_{0}+2 l_{1}+\cdots+2^{j}} l_{j} q^{k}=h_{j}\right\} .
$$


The inequality

$$
j M+M-1+l_{0}+2 l_{1}+\cdots+2^{j} l_{j}<(j+1) M+l_{0}+2 l_{1}+\cdots+2^{j+1} l_{j+1}
$$

for any $j \in\{0,1, \ldots, d-1\}$ implies that

$$
v\left(\bigcap_{j=0}^{d} t^{l_{0}+2 l_{1}+\cdots+2^{j} l_{j}} A_{j}\right)=q^{-(d+1) M} .
$$

It follows that $(\mu \times v)\left(\pi^{-1}(T)\right)=q^{-(d+1) M}$. Assertion (i) is proved.

Assertion (ii) follows from the definition of $\pi(\cdot)_{j}$. Assertion (ii) implies that for a.e. $\lambda \in X \times Y$, the element $\pi(t \lambda)$ belongs to $\mathcal{R}_{0} \pi(\lambda)$. The action of $H$ on $(Y, v)$ generates the odometer relation on $(Y, \nu)$ so that two elements $\left(y_{n}\right)_{n},\left(y_{n}^{\prime}\right)_{n} \in Y$ are equivalent if and only if for all but finitely many $n \in \mathbb{Z}$, we have $y_{n}=y_{n}^{\prime}$. The action of $H$ on $(X, \mu)$ is trivial. By the definition of $\pi$, for a.e. $\lambda \in X \times Y$, we have $\pi(H \lambda) \subset \mathcal{R}_{0} \pi(\lambda)$. Assertion (iii) is proved.

For $j \in \mathbb{N}$, we set

$$
\begin{aligned}
C_{j} & =\left\{\left(c_{i}\right)_{i \in \mathbb{N}} \in S \mid c_{j}=0\right\} \in \mathcal{B}_{S}, \\
B_{j} & =\pi^{-1}\left(C_{j}\right) \times Z \in \mathcal{B}_{W} .
\end{aligned}
$$

For any $j \in \mathbb{N}$, we then have $\omega\left(B_{j}\right)=\sigma\left(C_{j}\right)=q^{-M}$. The sequence $\left\{C_{j}\right\}_{j \in \mathbb{N}}$ is an a.i. sequence for the odometer relation $\mathcal{R}_{0}$. It follows from Lemma 2.1 and Lemma 4.2 (i), (iii) that $\left\{B_{j}\right\}_{j \in \mathbb{N}}$ is an a.i. sequence for the action $\Gamma \curvearrowright(W, \omega)$. We define $U_{j} \in[\mathcal{R}(\Gamma \curvearrowright W)]$ so that for any $l_{0}, l_{1}, \ldots, l_{j} \in\{0,1\}$, we have

$$
U_{j}=t^{l_{0}+2 l_{1}+\cdots+2^{j} l_{j}} a^{r q^{j M}} t^{-l_{0}-2 l_{1}-\cdots-2^{j} l_{j}}=a^{r q^{j M+l_{0}+2 l_{1}+\cdots+2^{j} l_{j}}}
$$

on the subset $X\left(l_{0}, l_{1}, \ldots, l_{j}\right) \times Y \times Z$ of $W$. Since $a$ acts on $X$ trivially, the map $U_{j}$ is indeed an element of the full group $[\mathcal{R}(\Gamma \curvearrowright W)]$.

Lemma 4.3. In the above notation, the following assertions hold:

(i) for any $j \in \mathbb{N}$, we have $U_{j} a=a U_{j}$ on $W$, and $U_{j} t=t U_{j}$ on the subset of $W$,

$$
(X \backslash X(\underbrace{1, \ldots, 1}_{j+1})) \times Y \times Z \text {; }
$$

(ii) for any $j \in \mathbb{N}$, we have $U_{j} B_{j} \cap B_{j}=\emptyset$. 
Proof. Assertion (i) follows from the definition of $U_{j}$. For any $j \in \mathbb{N}$, we have

$$
B_{j}=\bigsqcup_{l=0}^{2^{j+1}-1}\left\{\left(\left(x_{n}\right)_{n},\left(y_{m}\right)_{m}, z\right) \in W \mid \sum_{n=0}^{j} 2^{n} x_{n}=l, \sum_{k=0}^{M-1} y_{j M+k+l} q^{k}=0\right\} .
$$

The equation

$$
\sum_{k=0}^{M-1} y_{j M+k+l} q^{k}=0
$$

is equivalent to the condition that

$$
y_{j M+k+l}=0 \quad \text { for any } k \in\{0,1, \ldots, M-1\} .
$$

Since we have chosen the number $M$ so that $q^{M}>r$ in the beginning of this subsection, for any $j \in \mathbb{N}$, we have

$$
U_{j} B_{j}=\bigsqcup_{l=0}^{2^{j+1}-1}\left\{\left(\left(x_{n}\right)_{n},\left(y_{m}\right)_{m}, z\right) \in W \mid \sum_{n=0}^{j} 2^{n} x_{n}=l, \sum_{k=0}^{M-1} y_{j M+k+l} q^{k}=r\right\} .
$$

Assertion (ii) follows.

Theorem 4.4. In the above notation, the sequence $\left\{U_{j}\right\}_{j \in \mathbb{N}}$ is a non-trivial a.c. sequence for the action $\Gamma \curvearrowright(W, \omega)$. The action $\Gamma \curvearrowright(W, \omega)$ is therefore stable.

Proof. It suffices to check the following three conditions:

(1) for any $B \in \mathcal{B}_{W}$, we have $\lim _{j} \omega\left(U_{j} B \triangle B\right)=0$;

(2) for any $g \in \Gamma$, we have $\lim _{j} \omega\left(\left\{w \in W \mid U_{j} g w \neq g U_{j} w\right\}\right)=0$;

(3) the sequence $\left\{B_{j}\right\}_{j \in \mathbb{N}}$ in $\mathcal{B}_{W}$ is an a.i. sequence for the action $\Gamma \curvearrowright(W, \omega)$, and we have $\omega\left(U_{j} B_{j} \triangle B_{j}\right)=2 q^{-M}$ for any $j \in \mathbb{N}$.

The element $a$ of $\Gamma$ acts on $(X, \mu)$ trivially. Since $a$ acts on $(Y, \nu)$ by the $q$-adic odometer, for any sequence $\left\{n_{k}\right\}_{k \in \mathbb{N}}$ of positive integers and any $A \in \mathcal{B}_{Y}$, we have

$$
\lim _{k \rightarrow \infty} v\left(a^{n_{k} q^{k}} A \triangle A\right)=0 .
$$

The convergence is uniform with respect to the sequence $\left\{n_{k}\right\}_{k \in \mathbb{N}}$. Combining this with Lemma 4.1 (ii), we obtain condition (1). Condition (2) follows from Lemma 4.3 (i). The former assertion in condition (3) has already been checked right after the proof of Lemma 4.2. Finally, the latter assertion follows from Lemma 4.3 (ii). 
4.3. The case of $p>1$. We assume $p>1$. Fix a positive integer $M$ with $(p q)^{M}>r$. We set

$$
S=\prod_{\mathbb{N}}\left\{0,1, \ldots,(p q)^{M}-1\right\} .
$$

We have constructed the action of $\Gamma$ on the standard probability space

$$
(Y, v)=\left(Y_{-}, v_{-}\right) \times\left(Y_{0}, v_{0}\right) \times\left(Y_{+}, v_{+}\right),
$$

where

$$
\begin{aligned}
Y_{-} & =\prod_{\mathbb{Z}_{-}}\{0,1, \ldots, q-1\}, \\
Y_{0} & =\prod_{\mathbb{N}}\{0,1, \ldots, p q-1\}, \\
Y_{+} & =\prod_{\mathbb{Z}_{+}}\{0,1, \ldots, p-1\} .
\end{aligned}
$$

Let $\Gamma$ act on $X \times Y$ diagonally. For each $j \in \mathbb{N}$, let $\theta_{j}: X \times Y \rightarrow\{0,1, \ldots, p q-1\}$ be the projection onto the $j$ th coordinate of $Y_{0}$.

We define a Borel map $\pi: X \times Y \rightarrow S$ as follows. Pick an element

$$
\lambda=\left(\left(x_{n}\right)_{n \in \mathbb{N}}, y\right) \in X \times Y .
$$

For each $j \in \mathbb{N}$, the $j$ th coordinate of $\pi(\lambda) \in S$, denoted by $\pi(\lambda)_{j}$, is defined by

$$
\pi(\lambda)_{j}=\sum_{k=0}^{M-1} \theta_{d_{j}+k}\left(t^{-x_{0}-2 x_{1}-\cdots-2^{j} x_{j}} \lambda\right)(p q)^{k},
$$

where we put $d_{j}=j+2^{j+1}$. For each $j \in \mathbb{N}$, we set

$$
\begin{aligned}
C_{j} & =\left\{\left(c_{i}\right)_{i \in \mathbb{N}} \in S \mid c_{j}=0\right\} \in \mathcal{B}_{S}, \\
B_{j} & =\pi^{-1}\left(C_{j}\right) \times Z \in \mathcal{B}_{W} .
\end{aligned}
$$

Lemma 4.5. In the above notation, the following assertions hold:

(i) for any $j \in \mathbb{N}$, we have $\omega\left(B_{j}\right)=(p q)^{-M}$;

(ii) the sequence $\left\{B_{j}\right\}_{j \in \mathbb{N}}$ in $\mathcal{B}_{W}$ is an a.i. sequence for the action $\Gamma \curvearrowright(W, \omega)$. 
Proof. Fix $j_{0} \in \mathbb{N}$. We set

$$
\Omega=X(\underbrace{0, \ldots, 0}_{j_{0}+1})
$$

and

$$
A=\bigcap_{k=0}^{M-1}\left\{\left(y_{-},\left(y_{i}\right)_{i \in \mathbb{N}}, y_{+}\right) \in Y_{-} \times Y_{0} \times Y_{+} \mid y_{d_{j_{0}}+k}=0\right\} .
$$

The equality $\pi^{-1}\left(C_{j_{0}}\right)=\bigsqcup_{l=0}^{2^{j_{0}+1}-1} t^{l}(\Omega \times A)$ then holds. Assertion (i) follows.

We prove assertion (ii). By the definition of $\pi$, for any $j \in \mathbb{N}$, we have $\pi(t \lambda)_{j}=\pi(\lambda)_{j}$ for any element $\lambda$ of the subset

$$
(X \backslash X(\underbrace{1, \ldots, 1}_{j+1})) \times Y
$$

of $X \times Y$. We therefore have $\lim _{j} \omega\left(t B_{j} \triangle B_{j}\right)=0$.

Let $\theta: Y \rightarrow Y_{0}$ be the projection. For $K \in \mathbb{N}$, we set

$$
Y(K)=\left\{y \in Y \mid \theta\left(t^{-k} a y\right)=\theta\left(t^{-k} y\right), \text { for all } k \geq K\right\} .
$$

Lemma 3.3 implies that $Y=\bigcup_{K \in \mathbb{N}} Y(K)$. Pick $\varepsilon>0$. Choose a number $K_{0} \in \mathbb{N}$ with $v\left(Y \backslash Y\left(K_{0}\right)\right)<\varepsilon / 2$. For $j \in \mathbb{N}$, we set

$$
X_{j}=\left\{\left(x_{n}\right)_{n \in \mathbb{N}} \in X \mid x_{0}+2 x_{1}+\cdots+2^{j} x_{j} \geq K_{0}\right\} .
$$

Choose $J \in \mathbb{N}$ such that, for any $j \in \mathbb{N}$ with $j \geq J$, we have $\mu\left(X \backslash X_{j}\right)<\varepsilon / 2$. For any $j \in \mathbb{N}$ and any $\lambda \in X_{j} \times Y\left(K_{0}\right)$, we have $\pi(a \lambda)_{j}=\pi(\lambda)_{j}$. For any $j \in \mathbb{N}$, the inclusion

$$
a\left(\pi^{-1}\left(C_{j}\right) \cap\left(X_{j} \times Y\left(K_{0}\right)\right)\right) \subset \pi^{-1}\left(C_{j}\right)
$$

thus holds. For any $j \in \mathbb{N}$ with $j \geq J$, we have

$$
\begin{aligned}
\omega\left(a B_{j} \backslash B_{j}\right) & =(\mu \times v)\left(a \pi^{-1}\left(C_{j}\right) \backslash \pi^{-1}\left(C_{j}\right)\right) \\
& \leq(\mu \times v)\left((X \times Y) \backslash\left(X_{j} \times Y\left(K_{0}\right)\right)\right) \\
& \leq \mu\left(X \backslash X_{j}\right)+v\left(Y \backslash Y\left(K_{0}\right)\right) \\
& <\varepsilon
\end{aligned}
$$

We therefore obtain $\lim _{j} \omega\left(a B_{j} \Delta B_{j}\right)=0$. Assertion (ii) is proved. 
For $j \in \mathbb{N}$, we define $U_{j} \in[\mathcal{R}(\Gamma \curvearrowright W)]$ so that, for any $l_{0}, l_{1}, \ldots, l_{j} \in\{0,1\}$,

$$
\begin{aligned}
U_{j} & =t^{l_{0}+2 l_{1}+\cdots+2^{j} l_{j}} a^{r(p q)^{d_{j}}} t^{-l_{0}-2 l_{1}-\cdots-2^{j} l_{j}} \\
& =a^{r p^{d_{j}-l_{0}-2 l_{1}-\cdots-2^{j} l_{j}} q^{d_{j}+l_{0}+2 l_{1}+\cdots+2^{j} l_{j}}}
\end{aligned}
$$

on the subset $X\left(l_{0}, l_{1}, \ldots, l_{j}\right) \times Y \times Z$ of $W$. Since $a$ acts on $X$ trivially, the map $U_{j}$ is indeed an element of the full group $[\mathcal{R}(\Gamma \curvearrowright W)]$.

Lemma 4.6. In the above notation, the following assertions hold:

(i) for any $j \in \mathbb{N}$, we have $U_{j} a=a U_{j}$ on $W$, and $U_{j} t=t U_{j}$ on the subset of $W$,

$$
(X \backslash X(\underbrace{1, \ldots, 1}_{j+1})) \times Y \times Z
$$

(ii) for any $j \in \mathbb{N}$, we have $U_{j} B_{j} \cap B_{j}=\emptyset$.

Proof. Assertion (i) follows from the definition of $U_{j}$. For any $j \in \mathbb{N}$, we have

$$
B_{j}=\bigsqcup_{l=0}^{2^{j+1}-1}\left\{(\lambda, z) \in W \mid \sum_{n=0}^{j} 2^{n} x_{n}=l, \sum_{k=0}^{M-1} \theta_{d_{j}+k}\left(t^{-l} \lambda\right)(p q)^{k}=0\right\},
$$

where we put $\lambda=\left(\left(x_{n}\right)_{n \in \mathbb{N}}, y\right) \in X \times Y$. Since we have chosen the number $M$ so that $(p q)^{M}>r$ in the beginning of this subsection, for any $j \in \mathbb{N}$, we have

$$
U_{j} B_{j}=\bigsqcup_{l=0}^{2^{j+1}-1}\left\{(\lambda, z) \in W \mid \sum_{n=0}^{j} 2^{n} x_{n}=l, \sum_{k=0}^{M-1} \theta_{d_{j}+k}\left(t^{-l} \lambda\right)(p q)^{k}=r\right\} .
$$

Assertion (ii) follows.

Theorem 4.7. In the above notation, the sequence $\left\{U_{j}\right\}_{j \in \mathbb{N}}$ is a non-trivial a.c. sequence for the action $\Gamma \curvearrowright(W, \omega)$. The action $\Gamma \curvearrowright(W, \omega)$ is therefore stable.

Proof. It suffices to check the following three conditions:

(1) for any $B \in \mathcal{B}_{W}$, we have $\lim _{j} \omega\left(U_{j} B \triangle B\right)=0$;

(2) for any $g \in \Gamma$, we have $\lim _{j} \omega\left(\left\{w \in W \mid U_{j} g w \neq g U_{j} w\right\}\right)=0$;

(3) the sequence $\left\{B_{j}\right\}_{j \in \mathbb{N}}$ in $\mathcal{B}_{W}$ is an a.i. sequence for the action $\Gamma \curvearrowright(W, \omega)$, and we have $\omega\left(U_{j} B_{j} \triangle B_{j}\right)=2(p q)^{-M}$ for any $j \in \mathbb{N}$. 
The element $a$ of $\Gamma$ acts on $(X, \mu)$ trivially. Since $a$ acts on $\left(Y_{0}, \nu_{0}\right)$ by the $(p q)$-adic odometer, and acts on both $\left(Y_{-}, \nu_{-}\right)$and $\left(Y_{+}, \nu_{+}\right)$trivially, for any sequence $\left\{n_{k}\right\}_{k \in \mathbb{N}}$ of positive integers and any $A \in \mathcal{B}_{Y}$,

$$
\lim _{k \rightarrow \infty} v\left(a^{n_{k}(p q)^{k}} A \triangle A\right)=0 .
$$

The convergence is uniform with respect to the sequence $\left\{n_{k}\right\}_{k \in \mathbb{N}}$. For any $j \in \mathbb{N}$, we have a Borel partition $X=\bigsqcup_{l=0}^{2^{j+1}-1} X_{l}$ such that' for any $l \in\{0,1, \ldots$, $\left.2^{j+1}-1\right\}$, the restriction of $U_{j}$ to $X_{l} \times Y \times Z$ is equal to a power of $a$, and its exponent is divisible by $r(p q)^{j}$ because $d_{j}-\left(2^{j+1}-1\right)>j$. Combining this with Lemma 4.1 (ii), we obtain condition (1). Condition (2) follows from Lemma 4.6 (i). The former assertion in condition (3) is proved in Lemma 4.5 (ii). The latter assertion follows from Lemma 4.5 (i) and Lemma 4.6 (ii).

Proof of Theorem 1.1. Let $p, q, r$ and $\Gamma=\mathrm{BS}(r p, r q)$ be the symbols introduced in the beginning of this section. It is enough to show that for any two integers $k$, $l$ with $r p=|k|$ and $r q=|l|$, the group BS $(k, l)$ has an ergodic, free, p.m.p. and stable action. We put $\Lambda=\mathrm{BS}(k, l)$. If $k l$ is positive, then $\Gamma$ and $\Lambda$ are isomorphic, and the desired assertion follows from Theorems 4.4 and 4.7. Assume that $k l$ is negative. There exists an index 2 subgroup of $\Gamma$ isomorphic to an index 2 subgroup of $\Lambda$. In fact, if we have the presentations

$$
\begin{aligned}
& \Gamma=\left\langle a, t \mid t a^{r p} t^{-1}=a^{r q}\right\rangle, \\
& \Lambda=\left\langle b, u \mid u b^{k} u^{-1}=b^{l}\right\rangle,
\end{aligned}
$$

then let $\Gamma_{1}$ denote the subgroup of $\Gamma$ generated by $a, t_{a} t^{-1}$ and $t^{2}$, and let $\Lambda_{1}$ denote the subgroup of $\Lambda$ generated by $b, u b u^{-1}$ and $u^{2}$. We then have $\left[\Gamma: \Gamma_{1}\right]=2$ and $\left[\Lambda: \Lambda_{1}\right]=2$. The homomorphism $\varphi$ from $\Gamma_{1}$ into $\Lambda_{1}$ with $\varphi(a)=b$, $\varphi\left(t a t^{-1}\right)=u b^{-1} u^{-1}$ and $\varphi\left(t^{2}\right)=u^{2}$ is well-defined and is an isomorphism.

Let $\Gamma \curvearrowright(W, \omega)$ be the action constructed in Theorems 4.4 and 4.7. The subset $W_{1}=X(0) \times Y \times Z$ is $\Gamma_{1}$-invariant and has $\omega$-measure $1 / 2$. We can define an action of $\Lambda$ on $(W, \omega)$ such that the associated equivalence relation is equal to that for the action $\Gamma \curvearrowright(W, \omega)$; the subset $W_{1}$ is $\Lambda_{1}$-invariant; and the actions $\Gamma_{1} \curvearrowright W_{1}$ and $\Lambda_{1} \curvearrowright W_{1}$ are conjugate through the isomorphism $\varphi$. This action $\Lambda \curvearrowright(W, \omega)$ is a desired one. 
4.4. Stable actions of $\boldsymbol{H}$. Let $p, q, r, \Gamma=\mathrm{BS}(r p, r q)$ and $H$ be the symbols introduced in the beginning of this section. Let $\Gamma \curvearrowright(Y, v)$ and $\Gamma \curvearrowright(Z, \xi)$ be the actions constructed in Sections 4.1-4.3. We set

$$
\left(W_{1}, \omega_{1}\right)=(Y, \nu) \times(Z, \xi)
$$

and define an action $H \curvearrowright\left(W_{1}, \omega_{1}\right)$ as the diagonal action, which is ergodic, p.m.p. and essentially free.

Theorem 4.8. In the above notation, the action $H \curvearrowright\left(W_{1}, \omega_{1}\right)$ is stable.

We construct a non-trivial a.c. sequence for this action. The argument is similar to and simpler than those in Sections 4.2 and 4.3.

Proof of Theorem 4.8 in the case of $p=1$. We define the positive integer $M$, the standard probability space $(S, \sigma)$ and the odometer relation $\mathcal{R}_{0}$ on $(S, \sigma)$ as in the beginning of Section 4.2. Define a Borel map $\tau: Y \rightarrow S$ as follows. Pick an element $y=\left(y_{n}\right)_{n \in \mathbb{Z}}$ of $Y$. For each $j \in \mathbb{N}$, the $j$ th coordinate of $\tau(y)$, denoted by $\tau(y)_{j}$, is defined by the formula

$$
\tau(y)_{j}=\sum_{k=0}^{M-1} y_{j M+k} q^{k} .
$$

The equality $\tau_{*} \nu=\sigma$ then holds. For a.e. $y \in Y$, we have $\tau(H y) \subset \mathcal{R}_{0} \tau(y)$ because the action $H \curvearrowright(Y, v)$ generates the odometer relation on $(Y, v)$.

For $j \in \mathbb{N}$, let $C_{j}=\left\{\left(c_{i}\right)_{i \in \mathbb{N}} \in S \mid c_{j}=0\right\}$ be the Borel subset of $S$ defined in Section 4.2. Set $A_{j}=\tau^{-1}\left(C_{j}\right) \times Z$. For any $j \in \mathbb{N}$, we have $\omega_{1}\left(A_{j}\right)=\sigma\left(C_{j}\right)=$ $q^{-M}$. The sequence $\left\{A_{j}\right\}_{j \in \mathbb{N}}$ is an a.i. sequence for the action $H \curvearrowright\left(W_{1}, \omega_{1}\right)$. For $j \in \mathbb{N}$, we define an element $V_{j}$ of $\left[\mathcal{R}\left(H \curvearrowright W_{1}\right)\right]$ by setting $V_{j}=a^{r q^{j M}}$ on $W_{1}$. The set $A_{j}$ consists of all points $(y, z)$ of $W_{1}=Y \times Z$ with $\tau(y)_{j}=0$. The set $V_{j} A_{j}$ consists of all points $(y, z)$ of $W_{1}$ with $\tau(y)_{j}=r$ because we assumed $q^{M}>r$. It follows that $V_{j} A_{j} \cap A_{j}=\emptyset$ and $\omega_{1}\left(V_{j} A_{j} \triangle A_{j}\right)=2 q^{-M}$ for any $j \in \mathbb{N}$.

We now check the following three conditions:

(1) for any $A \in \mathcal{B}_{W_{1}}$, we have $\lim _{j} \omega_{1}\left(V_{j} A \triangle A\right)=0$;

(2) for any $g \in H$, we have $\lim _{j} \omega_{1}\left(\left\{w \in W_{1} \mid V_{j} g w \neq g V_{j} w\right\}\right)=0$;

(3) the sequence $\left\{A_{j}\right\}_{j \in \mathbb{N}}$ in $\mathcal{B}_{W_{1}}$ is an a.i. sequence for the action $H \curvearrowright\left(W_{1}, \omega_{1}\right)$, and we have $\omega_{1}\left(V_{j} A_{j} \triangle A_{j}\right)=2 q^{-M}$ for any $j \in \mathbb{N}$. 
Condition (1) follows from Lemma 4.1 (ii) and that $a$ acts on $(Y, v)$ by the $q$-adic odometer. Condition (2) holds because for any $g \in H$, we have $g a^{r q^{k}} g^{-1}=a^{r q^{k}}$ for any sufficiently large, positive integer $k$. Condition (3) has already been checked in the previous paragraph. We have shown that $\left\{V_{j}\right\}_{j \in \mathbb{N}}$ is a non-trivial a.c. sequence for the action $H \curvearrowright\left(W_{1}, \omega_{1}\right)$. Theorem 4.8 in the case of $p=1$ is proved.

Proof of Theorem 4.8 in the case of $p>1$. The proof of this case is similar to that in the case of $p=1$. We hence give only a sketch of the proof. Let $M$ be a positive integer with $(p q)^{M}>r$, and define the standard Borel space

$$
S=\prod_{\mathbb{N}}\left\{0,1, \ldots,(p q)^{M}-1\right\}
$$

as in the beginning of Section 4.3. Let $\sigma$ be the probability measure on $S$ defined as the direct product of the uniformly distributed probability measure on the set $\left\{0,1, \ldots,(p q)^{M}-1\right\}$. Let $\mathcal{R}_{0}$ denote the odometer relation on $(S, \sigma)$ so that two elements $\left(c_{i}\right)_{i},\left(c_{i}^{\prime}\right)_{i} \in S$ are equivalent if and only if for any sufficiently large $i \in \mathbb{N}$, we have $c_{i}=c_{i}^{\prime}$.

Define a Borel map

$$
\tau: Y \longrightarrow S
$$

as follows. Pick an element $y=\left(y_{-},\left(y_{i}\right)_{i \in \mathbb{N}}, y_{+}\right)$of $Y=Y_{-} \times Y_{0} \times Y_{+}$. For each $j \in \mathbb{N}$, the $j$ th coordinate of $\tau(y)$, denoted by $\tau(y)_{j}$, is defined by the formula

$$
\tau(y)_{j}=\sum_{k=0}^{M-1} y_{j M+k}(p q)^{k} .
$$

The equality $\tau_{*} \nu=\sigma$ then holds. For a.e. $y \in Y$, we have $\tau(H y) \subset \mathcal{R}_{0} \tau(y)$ because the action $H \curvearrowright(Y, v)$ generates the equivalence relation $\mathcal{S}_{0}$ on $(Y, v)$ defined right before Remark 3.2.

For $j \in \mathbb{N}$, we set $C_{j}=\left\{\left(c_{i}\right)_{i \in \mathbb{N}} \in S \mid c_{j}=0\right\}$ and $A_{j}=\tau^{-1}\left(C_{j}\right) \times Z$. We define an element $V_{j}$ of $\left[\mathcal{R}\left(H \curvearrowright W_{1}\right)\right]$ by $V_{j}=a^{r(p q)^{j M}}$ on $W_{1}$. The sequence $\left\{A_{j}\right\}_{j \in \mathbb{N}}$ is then an a.i. sequence for the action $H \curvearrowright\left(W_{1}, \omega_{1}\right)$. We have $V_{j} A_{j} \cap A_{j}=\emptyset$ and $\omega_{1}\left(V_{j} A_{j} \triangle A_{j}\right)=2(p q)^{-M}$ for any $j \in \mathbb{N}$. The sequence $\left\{V_{j}\right\}_{j \in \mathbb{N}}$ can be checked to be a non-trivial a.c. sequence for the action $H \curvearrowright\left(W_{1}, \omega_{1}\right)$ along the proof in the case of $p=1$.

Remark 4.9. Let $k$ and $l$ be integers with $r p=|k|$ and $r q=|l|$. Set

$$
\Lambda=\mathrm{BS}(k, l)=\left\langle b, u \mid u b^{k} u^{-1}=b^{l}\right\rangle .
$$

The normal subgroup of $\Lambda$ generated by $b$ is isomorphic to $H$ and therefore has an ergodic, free, p.m.p. and stable action by Theorem 4.8 . 


\section{Semi-direct products ME to direct products}

Definition 5.1. Two discrete groups $\Gamma$ and $\Lambda$ are called measure equivalent (ME) if there exists a measure-preserving action of $\Gamma \times \Lambda$ on a standard Borel space $\Sigma$ with a $\sigma$-finite positive measure $m$ such that we have Borel subsets $X, Y$ of $\Sigma$ with $m(X)<\infty, m(Y)<\infty$ and the equality $\Sigma=\bigsqcup_{\gamma \in \Gamma}(\gamma, e) Y=\bigsqcup_{\lambda \in \Lambda}(e, \lambda) X$ up to $m$-null sets.

ME is indeed an equivalence relation between discrete groups (see [8, Section 2]). It is known that two discrete groups $\Gamma$ and $\Lambda$ are ME if and only if there exist ergodic, essentially free and p.m.p. actions $\Gamma \curvearrowright(X, \mu)$ and $\Lambda \curvearrowright(Y, v)$ which are weakly orbit equivalent, that is, we have Borel subsets $A \subset X$ and $B \subset Y$ with positive measure such that the two equivalence relations

$$
\mathcal{R}(\Gamma \curvearrowright X) \cap(A \times A) \text { and } \mathcal{R}(\Lambda \curvearrowright Y) \cap(B \times B)
$$

are isomorphic (see [9, Section 3]). To prove Theorem 1.2, we need the following:

Lemma 5.2. Let $K, L$ and $M$ be discrete groups. Let $G=(K \times L) \rtimes M$ be a semi-direct product such that for any $m \in M$, we have $m(K \times\{e\}) m^{-1}=K \times\{e\}$ and $m(\{e\} \times L) m^{-1}=\{e\} \times L$. Suppose that we have a non-increasing sequence of finite index, normal subgroups of $M, M=M_{0}>M_{1}>M_{2}>\cdots$, with $K$ equal to the union of the centralizer of $M_{n}$ in $K$ for $n \in \mathbb{N}$.

We define $H=K \times(L \rtimes M)$ as the direct product of $K$ with the subgroup $L \rtimes M$ of $G$. Then $G$ and $H$ are $M E$.

Proof. Pick free and p.m.p. actions $K \rtimes M \curvearrowright(X, \mu)$ and $L \rtimes M \curvearrowright(Y, \nu)$ of the subgroups (or quotients) of $G$. We define $Z$ as the projective $\operatorname{limit} \lim M / M_{n}$ and

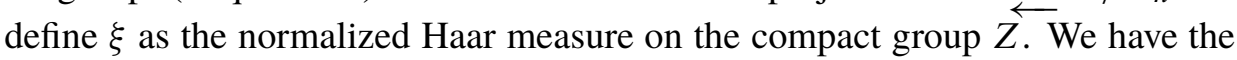
p.m.p. action of $M$ on $(Z, \xi)$ defined by left multiplication. We define an action of $G$ on the direct product $(W, \omega)=(X, \mu) \times(Y, \nu) \times(Z, \xi)$ by the formula

$$
((k, l), m)(x, y, z)=((k, m) x,(l, m) y, m z)
$$

for $k \in K, l \in L, m \in M, x \in X, y \in Y$ and $z \in Z$. This action of $G$ is free and p.m.p.

For each $n \in \mathbb{N}$, let $K_{n}$ denote the centralizer of $M_{n}$ in $K$ as subgroups of $G$. Let $\pi_{n}: Z \rightarrow M / M_{n}$ be the canonical projection. We define an action of $K_{n}$ on $(W, \omega)$, denoted by $\alpha_{n}$, by the formula $\alpha_{n}(k) w=m k^{-1} w$ for $k \in K_{n}, m \in M$ and $w \in X \times Y \times \pi_{n}^{-1}\left(m M_{n}\right)$. This action is well-defined and commutes with the action of $M$ on $(W, \omega)$. For any $n \in \mathbb{N}$ and any $k \in K_{n}$, we have $\alpha_{n+1}(k)=\alpha_{n}(k)$. 
By assumption, $K$ is equal to the union $\bigcup_{n} K_{n}$. We can thus define an action of $K$ on $(W, \omega)$, denoted by $\alpha$, by $\alpha(k) w=\alpha_{n}(k) w$ for $k \in K_{n}$ and $w \in W$. This action $\alpha$ of $K$ commutes with the action of the subgroup $L \rtimes M$ on $(W, \omega)$. We therefore obtain an action of $H$ on $(W, \omega)$. This action is free and p.m.p., and gives rise to the same equivalence relation as that for the action of $G$ on $(W, \omega)$. It follows that $G$ and $H$ are ME.

Proof of Theorem 1.2. Applying an argument of the same kind as in the proof of Theorem 1.1, we can reduce the proof of Theorem 1.2 to that in the case where $p$ and $q$ are integers with $2 \leq p<q$. Let $p$ and $q$ be such integers. Let $S$ be a set of prime numbers dividing neither $p$ nor $q$. We may assume that $S$ is nonempty. We set $F=\mathbb{Z}_{S}$ and define $\Lambda$ as the HNN extension of $F$ relative to the isomorphism $\alpha$ from $p F$ onto $q F$ multiplying by $q / p$. Let $t$ denote the element of $\Lambda$ implementing $\alpha$ with the relation $t b t^{-1}=\alpha(b)$ for any $b \in p F$. We will construct a locally compact and second countable group $G$ containing a lattice isomorphic to $\Lambda$.

Let $T$ denote the Bass-Serre tree associated with the decomposition of $\Lambda$ into the HNN extension. The set of vertices of $T$ is $\Lambda / F$, and the set of edges of $T$ is $\Lambda /(q F)$. For $\lambda \in \Lambda$, the edge corresponding to $\lambda(q F) \in \Lambda /(q F)$ joins the vertices corresponding to $\lambda F, \lambda t F \in \Lambda / F$. We introduce an orientation of $T$ so that for each $\lambda \in \Lambda$, the vertex corresponding to $\lambda F$ is the origin of the edge corresponding to $\lambda(q F)$. The group $\Lambda$ acts on $T$ by left multiplication, as simplicial automorphisms preserving this orientation. Let $\operatorname{Aut}(T)$ denote the group of orientation-preserving simplicial automorphisms of $T$, which is locally compact and second countable. We have the homomorphism $\imath: \Lambda \rightarrow \operatorname{Aut}(T)$ associated with the action of $\Lambda$ on $T$. We also have the continuous homomorphism $\pi: \operatorname{Aut}(T) \rightarrow \mathbb{Z}$ with $\pi(l(t))=1$ and $\pi(\varphi)=0$ for any element $\varphi$ of $\operatorname{Aut}(T)$ fixing a vertex of $T$.

For a prime number $r$, let $Q_{r}$ denote the quotient group $\mathbb{Q}_{r} / \mathbb{Z}_{r}$, which is regarded as a discrete group. We define $Q$ as the direct sum $\bigoplus_{r \in S} Q_{r}$. For each $r \in S$, since neither $p$ nor $q$ is divisible by $r$, the multiplication by $q / p$ induces an automorphism of $Q_{r}$. We define an action of $\operatorname{Aut}(T)$ on $Q \times \mathbb{R}$ by automorphisms, by the formula

$$
\varphi\left(\left(x_{r}\right)_{r \in S}, y\right)=\left(\left((q / p)^{\pi(\varphi)} x_{r}\right)_{r \in S},(q / p)^{\pi(\varphi)} y\right)
$$

for $\varphi \in \operatorname{Aut}(T),\left(x_{r}\right)_{r \in S} \in Q$ and $y \in \mathbb{R}$. Let $G$ denote the associated semi-direct product $(Q \times \mathbb{R}) \rtimes \operatorname{Aut}(T)$. 
We define a homomorphism $\tau: \Lambda \rightarrow G$ by

$$
\tau(b)=\left(\left(\left([b]_{r}\right)_{r \in S}, b\right), l(b)\right), \quad \tau(t)=((0,0), l(t))
$$

for any $b \in F$, where $[b]_{r}$ denotes the equivalence class of $b$ in $Q_{r}$ for $r \in S$. The homomorphism $\tau$ is injective. The image $\tau(\Lambda)$ is a lattice in $G$ with $\{0\} \times[0,1) \times K_{0}$ a fundamental domain, where $K_{0}$ is the stabilizer in $\operatorname{Aut}(T)$ of the vertex of $T$ whose stabilizer in $\Lambda$ is equal to $F$.

Let $E$ be the infinite cyclic subgroup of $F$ generated by the multiplicative unit of the ring $F$. We define $\Gamma$ as the subgroup of $\Lambda$ generated by $E$ and $t$. The group $\Gamma$ is the HNN extension generated by $E$ and $t$ and whose relations are $t b t^{-1}=\alpha(b)$ for any $b \in p E$. It follows that $\Gamma$ is isomorphic to $\operatorname{BS}(p, q)$. The image $\tau(\Gamma)$ is a lattice in the subgroup $(\{0\} \times \mathbb{R}) \rtimes \operatorname{Aut}(T)$ of $G$, with $\{0\} \times[0,1) \times K_{0}$ a fundamental domain. Let $\Delta$ be the subgroup of $G$ generated by $Q$ and $\tau(\Gamma)$. The group $\Delta$ is written as the semi-direct product

$$
(Q \times \imath(\operatorname{ker}(\pi \circ \imath) \cap \Gamma)) \rtimes\langle l(t)\rangle
$$

and is a lattice in $G$ with $\{0\} \times[0,1) \times K_{0}$ a fundamental domain. It turns out that $\Lambda$ and $\Delta$ are ME. Since $Q$ is an increasing union of finite subgroups invariant under the action of $l(t)$, by applying Lemma 5.2, we see that $\Delta$ and $Q \times \Gamma$ are ME. The groups $\Lambda$ and $Q \times \Gamma$ are therefore ME. By Theorem 1.1, $\Lambda$ and $\Gamma$ are ME.

We end this section with another application of Lemma 5.2 and a comment on Monod-Shalom's class $\mathcal{C}$. Let $p$ and $q$ be coprime integers with $1 \leq p<q$. Let $r$ be a positive integer with $r p \geq 2$. Define the group $G=G(p, q)$ as in the beginning of Section 3. Set

$$
\Gamma=\operatorname{BS}(r p, r q)=\left\langle a, t \mid t a^{r p} t^{-1}=a^{r q}\right\rangle .
$$

We define the homomorphism $\epsilon: \Gamma \rightarrow G$ and the subgroups $H, N$ of $\Gamma$ as in the beginning of Section 4. We set $E=\langle a\rangle$ and define $L$ as the subgroup $\epsilon^{-1}(\epsilon(E))$ of $H$, which is the semi-direct product $N \rtimes\langle a\rangle$.

Proposition 5.3. Let $F_{\infty}$ denote the free group of countably infinite rank. Then $L$ and $F_{\infty} \times \mathbb{Z}$ are $M E$.

Proof. The group $N$ is free because it acts on the Bass-Serre tree associated with $\Gamma$ freely. For $k \in \mathbb{N}$, we denote by $Z_{k}$ the centralizer of $a^{r(p q)^{k}}$ in $N$. We then have the equality $N=\bigcup_{k \in \mathbb{N}} Z_{k}$. Since this union is strictly increasing, the group $N$ is not finitely generated. It turns out that $N$ is isomorphic to $F_{\infty}$. The proposition follows from Lemma 5.2. 
Remark 5.4. Monod-Shalom [18] introduced Class $e$ consisting of all discrete groups $A$ such that for some mixing unitary representation $\pi$ of $A$ on a Hilbert space, the second bounded cohomology group of $A$ with coefficient $\pi$ is non-zero. They proved the following three assertions:

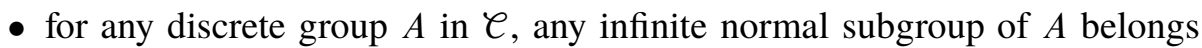
to $e$;

- whether a discrete group belongs to $\ell$ or not is invariant under $\mathrm{ME}$;

- no amenable group belongs to $\ell$.

We refer to [18, Proposition 7.4], [18, Corollary 7.6] and [18, Proposition 7.10 (i)] for these assertions, respectively. Combining these results with Proposition 5.3, we see that none of $L, H$ and $\Gamma$ belongs to $\ell$. Although the above Monod-

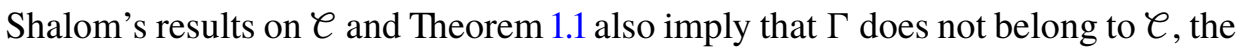
proof of this fact through Proposition 5.3 is much simpler.

\section{Stable actions of Vaes groups}

The following construction of the group $G$ is a slight generalization of the original one due to Vaes [23] (see Remark 6.2 for the group discussed in [23]).

Construction of a group. We follow the notation in [23]. For $n \in \mathbb{N}$, let $H_{n}$ be a non-trivial finite group, and let $E_{n}$ be a discrete group. Let $\Lambda$ be a discrete group acting on $H_{n}$ by automorphisms for each $n \in \mathbb{N}$. We denote this action as $\lambda \cdot h$ for $\lambda \in \Lambda$ and $h \in H_{n}$, using a dot. Set $K=\bigoplus_{n \in \mathbb{N}} H_{n}$. Let $\Lambda$ act on $K$ diagonally, that is, for $\lambda \in \Lambda$ and $h=\left(h_{n}\right)_{n \in \mathbb{N}} \in K$, we have $\lambda \cdot h=\left(\lambda \cdot h_{n}\right)_{n \in \mathbb{N}}$. For each $N \in \mathbb{N}$, let $K_{N}$ be the subgroup of $K$ defined by $K_{N}=\bigoplus_{n=N}^{\infty} H_{n}$. We set $G_{0}=K \rtimes \Lambda$ and inductively define a group $G_{N+1}$ as the amalgamated free product

$$
G_{N+1}=G_{N} *_{K_{N}}\left(K_{N} \times E_{N}\right),
$$

where $K_{N}$ is regarded as a subgroup of $G_{N}$ through the inclusion $K_{N}<K<$ $G_{0}<G_{N}$, and $G_{N}$ is naturally a subgroup of $G_{N+1}$. Let $G$ denote the inductive limit of the increasing sequence of groups, $G_{0}<G_{1}<G_{2}<\cdots$.

For any $N \in \mathbb{N}$, we have the homomorphism $\epsilon_{N}: G_{N+1} \rightarrow G_{N}$ that is the identity on $G_{N}$ and sends $E_{N}$ to the neutral element. We thus obtain the homomorphism $\epsilon: G \rightarrow G_{0}$ that is equal to $\epsilon_{0} \circ \epsilon_{1} \circ \cdots \circ \epsilon_{N}$ on $G_{N+1}$ for any $N \in \mathbb{N}$. Let $\delta: G \rightarrow \Lambda$ denote the composition of $\epsilon$ and the quotient map from $G_{0}$ onto $\Lambda$. 
Construction of an action The construction is similar to that in Section 4. For each $n \in \mathbb{N}$, let $\Lambda_{n}$ denote the subgroup of $\Lambda$ consisting of all elements acting on $H_{i}$ trivially for any $i \in \mathbb{N}$ with $i \leq n$. We define $X$ as the projective limit $\lim \Lambda / \Lambda_{n}$ and define $\mu$ as the normalized Haar measure on the compact group $X$. We have the action $\Lambda \curvearrowright(X, \mu)$ defined by left multiplication. Let $G$ act on $(X, \mu)$ through the homomorphism $\delta: G \rightarrow \Lambda$.

We set $Z_{0}=\prod_{n \in \mathbb{N}} H_{n}$ and denote by $\xi_{0}$ the normalized Haar measure on the compact group $Z_{0}$. Let $K$ act on $Z_{0}$ by left multiplication. We set

$$
(Z, \xi)=\prod_{G / K}\left(Z_{0}, \xi_{0}\right)
$$

and define a p.m.p. action of $G$ on $(Z, \xi)$ as the action co-induced from the action of $K$ on $\left(Z_{0}, \xi_{0}\right)$. Since the action $K \curvearrowright\left(Z_{0}, \xi_{0}\right)$ is essentially free, so is the action $G \curvearrowright(Z, \xi)$. If $\operatorname{ker} \epsilon$ is infinite, then the action $\operatorname{ker} \epsilon \curvearrowright(Z, \xi)$ is ergodic because ker $\epsilon$ acts on $G / K$ freely.

We set $(Y, v)=\left(Z_{0}, \xi_{0}\right)$. The group $\Lambda$ acts on the group $Y$ by automorphisms, and $K$ acts on $Y$ by left multiplication. We then obtain the action $G_{0} \curvearrowright(Y, v)$. Let $G$ act on $(Y, v)$ through the homomorphism $\epsilon: G \rightarrow G_{0}$.

We set

$$
(W, \omega)=(X, \mu) \times(Y, \nu) \times(Z, \xi)
$$

and define a p.m.p. action $G \curvearrowright(W, \omega)$ as the diagonal action so that for $g \in G$ and $w=(x, y, z) \in W$, we have $g w=(g x, g y, g z)$. The action $G \curvearrowright(W, \omega)$ is essentially free, and is ergodic if $\operatorname{ker} \epsilon$ is infinite.

Theorem 6.1. In the above notation, we assume that $\operatorname{ker} \epsilon$ is infinite. Then the action $G \curvearrowright(W, \omega)$ is stable.

Proof. For each $n \in \mathbb{N}$, let $\tau_{n}: X \rightarrow \Lambda / \Lambda_{n}$ be the canonical projection. We define a Borel map $\pi: W \rightarrow Y$ as follows. Pick $w=(x, y, z) \in W$ and $n \in \mathbb{N}$. Choosing $\lambda \in \Lambda$ with $x \in \tau_{n}^{-1}\left(\lambda \Lambda_{n}\right)$, we define the $n$th coordinate of $\pi(w)$, denoted by $\pi(w)_{n}$, as $\pi(w)_{n}=\lambda^{-1} \cdot y_{n}$, where $y_{n}$ denotes the $n$th coordinate of $y$. This is well-defined because $\Lambda_{n}$ acts on $H_{n}$ trivially. We can check the following:

(i) the equality $\pi_{*} \omega=v$ holds;

(ii) the map $\pi: W \rightarrow Y$ is $\Lambda$-invariant and (ker $\epsilon$ )-invariant;

(iii) for any $w \in W$, we have $\pi(K w)=K \pi(w)$. 
We now construct a non-trivial a.c. sequence for the action $G \curvearrowright(W, \omega)$. For each $n \in \mathbb{N}$, fix a non-neutral element $h_{n}$ of $H_{n}$. We choose a subset $I_{n}$ of $H_{n}$ with $h_{n} I_{n} \cap I_{n}=\emptyset$ and $\left|I_{n}\right| \geq\left|H_{n}\right| / 3$. Such an $I_{n}$ is obtained as follows. Let $M$ be the least integer with $M \geq\left|H_{n}\right| / 3$. We define elements of $H_{n}, l_{1}, \ldots, l_{M}$, inductively. Pick an arbitrary element $l_{1}$ of $H_{n}$. Let $m$ be a positive integer with $m<M$. If $l_{1}, \ldots, l_{m}$ are defined, then we set $L_{m}=\left\{l_{1}, \ldots, l_{m}\right\}$. The set

$$
H_{n} \backslash\left(h_{n}^{-1} L_{m} \cup L_{m} \cup h_{n} L_{m}\right)
$$

is non-empty because $m<\left|H_{n}\right| / 3$. Let $l_{m+1}$ be an element of this set. We defined $l_{1}, \ldots, l_{M} \in H_{n}$, and set $I_{n}=\left\{l_{1}, \ldots, l_{M}\right\}$. This is a desired set.

We set

$$
\begin{aligned}
& C_{n}=\left\{\left(y_{m}\right)_{m \in \mathbb{N}} \in Y \mid y_{n} \in I_{n}\right\} \in \mathcal{B}_{Y}, \\
& B_{n}=\pi^{-1}\left(C_{n}\right) \in \mathcal{B}_{W} .
\end{aligned}
$$

Define an element $U_{n}$ of $[\mathcal{R}(G \curvearrowright W)]$ by $U_{n}=\lambda h_{n} \lambda^{-1}$ on $\tau_{n}^{-1}\left(\lambda \Lambda_{n}\right) \times Y \times Z$ with $\lambda \in \Lambda$. This is well-defined because $\Lambda_{n}$ acts on $H_{n}$ trivially. We check the following three conditions:

(1) for any $A \in \mathcal{B}_{W}$, we have $\lim _{n} \omega\left(U_{n} A \triangle A\right)=0$;

(2) for any $g \in G$, we have $\lim _{n} \omega\left(\left\{w \in W \mid U_{n} g w \neq g U_{n} w\right\}\right)=0$;

(3) the sequence $\left\{B_{n}\right\}_{n \in \mathbb{N}}$ in $\mathcal{B}_{W}$ is an a.i. sequence for the action $G \curvearrowright(W, \omega)$, and we have $\omega\left(U_{n} B_{n} \triangle B_{n}\right) \geq 2 / 3$ for any $n \in \mathbb{N}$.

Let $\left\{\gamma_{n}\right\}_{n \in \mathbb{N}}$ be a sequence with $\gamma_{n} \in H_{n}$ for any $n \in \mathbb{N}$. Pick $g \in G$ and choose $N \in \mathbb{N}$ with $g \in G_{N}$. For any $n \in \mathbb{N}$ with $n \geq N-1$, we have $g^{-1} \gamma_{n} g \in H_{n}$. We therefore have $\lim _{n} \xi\left(\gamma_{n} D \triangle D\right)=0$ for any $D \in \mathcal{B}_{Z}$. By the definition of the action of $K$ on $Y$, we have $\lim _{n} v\left(\gamma_{n} C \triangle C\right)=0$ for any $C \in \mathcal{B}_{Y}$. These convergences are uniform with respect to the sequence $\left\{\gamma_{n}\right\}_{n \in \mathbb{N}}$. The action of $K$ on $X$ is trivial. Condition (1) follows.

For any $n \in \mathbb{N}$, by definition, $U_{n}$ commutes with any element of $\Lambda$. For any $m \in \mathbb{N}$, if $n \in \mathbb{N}$ is bigger than $m$, then $U_{n}$ commutes with any element of $H_{m}$ and $E_{m}$. Since $G$ is generated by $\Lambda, H_{m}$ and $E_{m}$ for all $m \in \mathbb{N}$, condition (2) follows.

The sequence $\left\{C_{n}\right\}_{n \in \mathbb{N}}$ in $\mathcal{B}_{Y}$ is an a.i. sequence for the action $K \curvearrowright(Y, v)$. Conditions (i)-(iii) and Lemma 2.1 imply that $\left\{B_{n}\right\}_{n \in \mathbb{N}}$ is an a.i. sequence for the action $G \curvearrowright(W, \omega)$. For any $n \in \mathbb{N}$, we have $\omega\left(U_{n} B_{n} \triangle B_{n}\right)=\left|h_{n} I_{n} \triangle I_{n}\right| /\left|H_{n}\right| \geq$ $2 / 3$. Condition (3) is proved.

Conditions (1)-(3) show that $\left\{U_{n}\right\}_{n \in \mathbb{N}}$ is a non-trivial a.c. sequence for the action $G \curvearrowright(W, \omega)$. The theorem follows from Theorem 2.2. 
Remark 6.2. Vaes [23] showed that $G$ is inner amenable, the conjugacy class of any non-neutral element of $G$ in $G$ is infinite, and the von Neumann algebra of $G$ does not have property Gamma, under the following assumption: We choose an arbitrary sequence of mutually distinct prime numbers, $\left\{p_{n}\right\}_{n \in \mathbb{N}}$. For each $n \in \mathbb{N}$, we set $H_{n}=\left(\mathbb{Z} / p_{n} \mathbb{Z}\right)^{3}$ and $E_{n}=\mathbb{Z}$, and set $\Lambda=S L(3, \mathbb{Z})$. The group $\Lambda$ naturally acts on $H_{n}$ by automorphisms. Theorem 6.1 therefore implies Theorem 1.3.

\section{References}

[1] S. Adams, Indecomposability of equivalence relations generated by word hyperbolic groups. Topology 33 (1994), no. 4, 785-798. Zbl 0838.20043 MR 1293310

[2] I. Chifan and C. Houdayer, Bass-Serre rigidity results in von Neumann algebras. Duke Math. J. 153 (2010), no. 1, 23-54. Zbl 1201.46057 MR 2641939

[3] I. Chifan and T. Sinclair, On the structural theory of $\mathrm{II}_{1}$ factors of negatively curved groups. Ann. Sci. Éc. Norm. Supér. (4) 46 (2013), no. 1, 1-33. Zbl 1290.46053 MR 3087388

[4] A. Connes, J. Feldman and B. Weiss, An amenable equivalence relation is generated by a single transformation. Ergodic Theory Dynamical Systems 1 (1981), no. 4, 431-450. Zbl 0491.28018 MR 0662736

[5] E. G. Effros, Property $\Gamma$ and inner amenability. Proc. Amer. Math. Soc. 47 (1975), 483-486. Zbl 0321.22011 MR 0355626

[6] J. Feldman and C. C. Moore, Ergodic equivalence relations, cohomology, and von Neumann algebras. I. Trans. Amer. Math. Soc. 234 (1977), no. 2, 289-324. Zbl 0369.22009 MR 0578656

[7] P. Fima, A note on the von Neumann algebra of a Baumslag-Solitar group. C. R. Math. Acad. Sci. Paris 349 (2011), no. 1-2, 25-27. Zbl 1218.46036 MR 2755690

[8] A. Furman, Gromov's measure equivalence and rigidity of higher rank lattices. Ann. of Math. (2) 150 (1999), no. 3, 1059-1081. Zbl 0943.22013 MR 1740986

[9] A. Furman, Orbit equivalence rigidity. Ann. of Math. (2) 150 (1999), no. 3, 1083-1108. Zbl 0943.22012 MR 1740985

[10] D. Gaboriau, Coût des relations d'équivalence et des groupes. Invent. Math. 139 (2000), no. 1, 41-98. Zbl 0939.28012 MR 1728876

[11] D. Gaboriau, Invariants $\ell^{2}$ de relations d'équivalence et de groupes. Publ. Math. Inst. Hautes Études Sci. 95 (2002), 93-150. Zbl 1022.37002 MR 1953191

[12] D. Gaboriau, Examples of groups that are measure equivalent to the free group. Ergodic Theory Dynam. Systems 25 (2005), no. 6, 1809-1827. Zbl 1130.37311 MR 2183295 
[13] C. Houdayer and S. Vaes, Type III factors with unique Cartan decomposition. J. Math. Pures Appl. (9) 100 (2013), no. 4, 564-590. Zbl 1291.46052 MR 3102166

[14] Y. Isono, Weak exactness for $C^{*}$-algebras and application to condition (AO). J. Funct. Anal. 264 (2013), no. 4, 964-998. Zbl 1284.46046 MR 3004955

[15] V. F. R. Jones and K. Schmidt, Asymptotically invariant sequences and approximate finiteness. Amer. J. Math. 109 (1987), no. 1, 91-114. Zbl 0638.28014 MR 0878200

[16] Y. Kida, The mapping class group from the viewpoint of measure equivalence theory. Mem. Amer. Math. Soc. 196 (2008), no. 916. Zbl 1277.37008 MR 2458794

[17] Y. Kida, Invariants of orbit equivalence relations and Baumslag-Solitar groups. Tohoku Math. J. (2) 66 (2014), no. 2, 205-258. Zbl 06341717 MR 3229595

[18] N. Monod and Y. Shalom, Orbit equivalence rigidity and bounded cohomology. Ann. of Math. (2) 164 (2006), no. 3, 825-878. Zbl 1129.37003 MR 2259246

[19] D. S. Ornstein and B. Weiss, Ergodic theory of amenable group actions. I. The Rohlin lemma. Bull. Amer. Math. Soc. (N.S.) 2 (1980), no. 1, 161-164. Zbl 0427.28018 MR 0551753

[20] N. Ozawa, A Kurosh type theorem for type $\mathrm{II}_{1}$ factors. Int. Math. Res. Not. 2006 (2006), article id. 97560, 21 pp. Zbl 1114.46041 MR 2211141

[21] S. Popa, On the superrigidity of malleable actions with spectral gap. J. Amer. Math. Soc. 21 (2008), no. 4, 981-1000. Zbl 1222.46048 MR 2425177

[22] Y. Stalder, Moyennabilité intérieure et extensions HNN. Ann. Inst. Fourier (Grenoble) 56 (2006), no. 2, 309-323. Zbl 1143.20013 MR 2226017

[23] S. Vaes, An inner amenable group whose von Neumann algebra does not have property Gamma. Acta Math. 208 (2012), no. 2, 389-394. Zbl 1250.46041 MR 2931384

[24] R. J. Zimmer, Ergodic actions of semisimple groups and product relations. Ann. of Math. (2) 118 (1983), no. 1, 9-19. Zbl 0545.22010 MR 0707158

Received September 21, 2012

Yoshikata Kida, Department of Mathematics, Kyoto University, 606-8502 Kyoto, Japan

e-mail:kida@math.kyoto-u.ac.jp 